\title{
Nobel Lecture: Bose-Einstein condensation in a dilute gas, the first 70 years and some recent experiments*
}

\author{
E. A. Cornell and C. E. Wieman \\ JILA, University of Colorado and National Institute of Standards and Technology, \\ and Department of Physics, University of Colorado, Boulder, Colorado 80309-0440
}

(Published 19 August 2002)

\begin{abstract}
Bose-Einstein condensation, or BEC, has a long and rich history dating from the early 1920s. In this article we will trace briefly over this history and some of the developments in physics that made possible our successful pursuit of BEC in a gas. We will then discuss what was involved in this quest. In this discussion we will go beyond the usual technical description to try and address certain questions that we now hear frequently, but are not covered in our past research papers. These are questions along the lines of: How did you get the idea and decide to pursue it? Did you know it was going to work? How long did it take you and why? We will review some our favorites from among the experiments we have carried out with BEC. There will then be a brief encore on why we are optimistic that BEC can be created with nearly any species of magnetically trappable atom. Throughout this article we will try to explain what makes BEC in a dilute gas so interesting, unique, and experimentally challenging. ${ }^{1}$
\end{abstract}

The notion of Bose statistics dates back to a 1924 paper in which Satyendranath Bose used a statistical argument to derive the black-body photon spectrum (Bose, 1924). Unable to publish his work, he sent it to Albert Einstein, who translated it into German and got it published. Einstein then extended the idea of Bose's counting statistics to the case of noninteracting atoms (Einstein, 1924, 1925). The result was Bose-Einstein statistics. Einstein immediately noticed a peculiar feature of the distribution of the atoms over the quantized energy levels predicted by these statistics. At very low but finite temperature a large fraction of the atoms would go into the lowest energy quantum state. In his words, "A separation is effected; one part condenses, the rest remains a saturated ideal gas" 2 (Einstein, 1925). This phenomenon we now know as Bose-Einstein condensation. The condition for this to happen is that the phase-space density must be greater than approximately unity, in natural units. Another way to express this is

\footnotetext{
*The 2001 Nobel Prize in Physics was shared by E. A. Cornell, Wolfgang Ketterle, and C. E. Wieman.

${ }^{1}$ This article is our "Nobel Lecture" and as such takes a relatively personal approach to the story of the development of experimental Bose-Einstein condensation. For a somewhat more scholarly treatment of the history, the interested reader is referred to E. A. Cornell, J. R. Ensher, and C. E. Wieman, "Experiments in dilute atomic Bose-Einstein condensation in Bose-Einstein Condensation in Atomic Gases," Proceedings of the International School of Physics "Enrico Fermi" Course $C X L$, edited by M. Inguscio, S. Stringari, and C. E. Wieman (Italian Physical Society, 1999), pp. 15-66, which is also available as cond-mat/9903109. For a reasonably complete technical review of the three years of explosive progress that immediately followed the first observation of BEC, we recommend reading the above article in combination with the corresponding review from Ketterle, cond-mat/9904034.

${ }^{2}$ English translation of Einstein's quotes and the historical interpretation are from Pais (1982), Subtle is the Lord... .
}

that the de Broglie wavelength, $\lambda_{\mathrm{dB}}$, of each atom must be large enough to overlap with its neighbor, or more precisely, $n \lambda_{\mathrm{dB}}^{3}>2.61$.

This prediction was not taken terribly seriously, even by Einstein himself, until Fritz London (1938) and Laszlo Tisza (1938) resurrected the idea in the mid 1930s as a possible mechanism underlying superfluidity in liquid helium 4. Their work was the first to bring out the idea of BEC displaying quantum behavior on a macroscopic size scale, the primary reason for much of its current attraction. Although it was a source of debate for decades, it is now recognized that the remarkable properties of superconductivity and superfluidity in both helium 3 and helium 4 are related to BEC, even though these systems are very different from the ideal gas considered by Einstein.

The appeal of the exotic behavior of superconductivity and of superfluidity, along with that of laser light, the third common system in which macroscopic quantum behavior is evident, provided much of our motivation in 1990 when we decided to pursue BEC in a gas. These three systems all have fascinating counterintuitive behavior arising from macroscopic occupation of a single quantum state. Any physicist would consider these phenomena among the most remarkable topics in physics. In 1990 we were confident that the addition of a new member to the family would constitute a major contribution to physics. (Only after we succeeded did we realize that the discovery of each of the original Macroscopic Three had been recognized with a Nobel Prize, and we are grateful that this trend has continued!) Although BEC shares the same underlying mechanism with these other systems, it seemed to us that the properties of BEC in a gas would be quite distinct. It is far more dilute and weakly interacting than liquid-helium superfluids, for example, but far more strongly interacting than the noninteracting light in a laser beam. Perhaps BEC's most distinctive feature (and this was not 
something we sufficiently appreciated, in 1990) is the ease with which its quantum wave function may be directly observed and manipulated. While neither of us was to read C. E. Hecht's prescient 1959 paper (Hecht, 1959) until well after we had observed BEC, we surely would have taken his concluding paragraph as our marching orders:

The suppositions of this note rest on the possibility of securing, say by atomic beam techniques, substantial quantities of electron-spin-oriented $\mathrm{H}, \mathrm{T}$ and $\mathrm{D}$ atoms. Although the experimental difficulties would be great and the relaxation behavior of such spin-oriented atoms essentially unknown, the possibility of opening a rich new field for the study of superfluid properties in both liquid and gaseous states would seem to demand the expenditure of maximum experimental effort. ${ }^{3}$

In any case, by 1990 we were awash in motivation. But this motivation would not have carried us far, had we not been able to take advantage of some key recent advances in science and technology, in particular, the progress in laser cooling and trapping and the extensive achievements of the spin-polarized-hydrogen community.

However, before launching into that story, it is perhaps worthwhile to reflect on just how exotic a system of indistinguishable particles truly is, and why BEC in a gas is such a daunting experimental challenge. It is easy at first to accept that two atoms can be so similar one to the other as to allow no possibility of telling them apart. However, confronting the physical implications of the concept of indistinguishable bosons can be troubling. For example, if there are ten bosonic particles to be arranged in two microstates of a system, the statistical weight of the configuration with ten particles in one state and zero in the other is exactly the same as the weight of the configuration with five particles in one state, five in the other. This 1:1 ratio of statistical weights is very counterintuitive and rather disquieting. The corresponding ratio for distinguishable objects, such as socks in drawers, that we observe every day is 1:252, profoundly different from 1:1. In the second of Einstein's two papers (Einstein, 1925; Pais, 1982) on BoseEinstein statistics, Einstein comments that "The... molecules are not treated as statistically independent..., and the differences between distinguishable and indistinguishable state counting ... express indirectly a certain hypothesis on a mutual influence of the molecules which for the time being is of a quite mysterious nature. This mutual influence is no less mysterious today, even though we can readily observe the variety of exotic behavior it causes such as the well-known enhanced probability for scattering into occupied states and, of course, Bose-Einstein condensation."

Not only does the Bose-Einstein phase transition offend our sensibilities as to how particles ought best to distribute themselves, it also runs counter to an unspo-

${ }^{3}$ Emphasis ours.

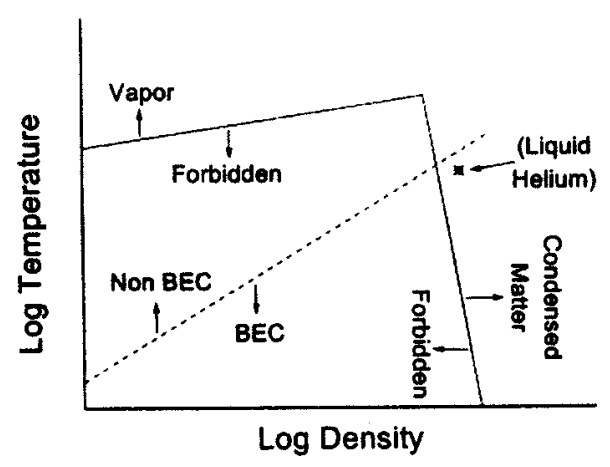

FIG. 1. Generic phase diagram common to all atoms: dotted line, the boundary between non-BEC and BEC; solid line, the boundary between allowed and forbidden regions of the temperature-density space. Note that at low and intermediate densities, BEC exists only in the thermodynamically forbidden regime.

ken assumption that a phase transition somehow involves thermodynamic stability. In fact, the regions immediately above and immediately below the transition in dilute-gas experiments are both deep in the thermodynamically forbidden regime. This point is best made by considering a qualitative phase diagram (Fig. 1), which shows the general features common to any atomic system. At low density and high temperature, there is a vapor phase. At high density there are various condensed phases. But the intermediate densities are thermodynamically forbidden, except at very high temperatures. The Bose-condensed region of the $n-T$ plane is utterly forbidden, except at such high densities that (with one exception) all known atoms or molecules would form a crystalline lattice, which would rule out Bose condensation. The single exception, helium, remains a liquid below the BEC transition. However, reaching BEC under dilute conditions (say, at densities 10 or 100 times lower than conventional liquid helium) is as thermodynamically forbidden to helium as it is to any other atom.

Of course, forbidden is not the same as impossible; indeed, to paraphrase an old Joseph Heller joke, if it were really impossible, they wouldn't have bothered to forbid it. It comes down in the end to differing time scales for different sorts of equilibrium. A gas of atoms can come into kinetic equilibrium via two-body collisions, whereas it requires three-body collisions to achieve chemical equilibrium (i.e., to form molecules and thence solids). At sufficiently low densities, the twobody rate will dominate the three-body rate, and a gas will reach kinetic equilibrium, perhaps in a metastable Bose-Einstein condensate, long before the gas finds its way to the ultimately stable solid-state condition. The need to maintain metastability usually dictates a more stringent upper limit on density than does the desire to create a dilute system. Densities around $10^{20} \mathrm{~cm}^{-3}$, for instance, would be a hundred times more dilute than a condensed-matter helium superfluid. But creating such a gas is quite impractical even at an additional factor-of1000 lower density, say $10^{17} \mathrm{~cm}^{-3}$, when metastability times would be on the order of a few microseconds; 
more realistic are densities on the order of $10^{14} \mathrm{~cm}^{-3}$. The low densities mandated by the need to maintain long-lived metastability in turn make necessary the achievement of still lower temperatures if one is to reach BEC.

Thus the great experimental hurdle that must be overcome to create BEC in a dilute gas is to form and keep a sample that is so deeply forbidden. Since our subsequent discussion will focus only on BEC in dilute gases, we shall refer to this simply as BEC in the sections below and avoid endlessly repeating "in a dilute gas."

Efforts to make a dilute BEC in an atomic gas were sparked by Stwalley and Nosanow (1976). They argued that spin-polarized hydrogen had no bound states and hence would remain a gas down to zero temperature, and so it would be a good candidate for BEC. This stimulated a number of experimental groups (Silvera and Walraven, 1980; Hardy et al., 1982; Hess et al., 1983; Johnson et al., 1984) in the late 1970s and early 1980s to begin pursuing this idea using traditional cryogenics to cool a sample of polarized hydrogen. Spin-polarized hydrogen was first stabilized by Silvera and Walraven in 1980 , and by the mid 1980s spin-polarized hydrogen had been brought within a factor of 50 of condensing (Hess et al., 1983). These experiments were performed in a dilution refrigerator, in a cell in which the walls were coated with superfluid liquid helium as a nonstick coating for the hydrogen. The hydrogen gas was compressed using a piston-in-cylinder arrangement (Bell et al., 1986) or inside a helium bubble (Sprik et al., 1985). These attempts failed, however, because when the cell was made very cold the hydrogen stuck to the helium surface and recombined. When one tried to avoid that problem by warming the cell sufficiently to prevent sticking, the density required to reach BEC was correspondingly increased, which led to another problem. The requisite densities could not be reached because the rate of threebody recombination of atoms into hydrogen molecules goes up rapidly with density and the resulting loss of atoms limited the density (Hess, 1986).

Stymied by these problems, Harold Hess (Hess, 1986) from the MIT hydrogen group realized that magnetic trapping of atoms (Migdall et al., 1985; Bagnato et al., 1987) would be an improvement over a cell. Atoms in a magnetic trap have no contact with a physical surface and thus the surface-recombination problem could be circumvented. Moreover, thermally isolated atoms in a magnetic trap would allow cooling by evaporation to far lower temperatures than previously obtained. In a remarkable paper, Hess (1986) laid out most of the important concepts of evaporative cooling of trapped atoms for the attainment of BEC. Let the highest-energy atoms escape from the trap, and the mean energy, and thus the temperature, of the remaining atoms will decrease. For a dilute gas in an inhomogeneous potential, decreasing the temperature will decrease the occupied volume. One can thus actually increase the density of the remaining atoms by removing atoms from the sample. The all important (for BEC) phase-space density is dramatically increased as this happens because density is rising while temperature is decreasing. The Cornell University hydrogen group also considered evaporative cooling (Lovelace et al., 1985). By 1988 the MIT group had demonstrated these virtues of evaporative cooling of magnetically trapped spin-polarized hydrogen. By 1991 they obtained, at a temperature of $100^{\circ} \mathrm{K}$, a density that was only a factor of 5 below BEC (Doyle, 1991a). Further progress was limited by dipolar relaxation, but perhaps more fundamentally by loss of signal-to-noise, and the difficulty of measuring the characteristics of the coldest and smallest clouds (Doyle, 1991b). Evaporative work was also performed by the Amsterdam group (Luiten et al., 1993).

At roughly the same time, but independent from the hydrogen work, an entirely different type of cold-atom physics and technology was being developed. Laser cooling and trapping has been reviewed elsewhere (Arimondo et al., 1991; Chu, 1998; Cohen-Tannoudji, 1998; Phillips, 1998), but here we mention some of the highlights most relevant to our work. The idea that laser light could be used to cool atoms was suggested in early papers by Wineland and Dehmelt (1975), by Hänsch and Schawlow (1975), and by Letokhov's group (Letokhov, 1968). Early optical force experiments were performed by Ashkin (Bjorkholm et al., 1978). Trapped ions were laser-cooled at the University of Washington (Neuhauser et al., 1978) and at the National Bureau of Standards (now NIST) in Boulder (Wineland et al., 1978). Atomic beams were deflected and slowed in the early 1980s (Andreev et al., 1981; Ertmer et al., 1985; Prodan et al., 1985). Optical molasses, where the atoms are cooled to very low temperatures by six perpendicular intersecting laser beams, was first studied at Bell Labs (Chu et al., 1985). Measured temperatures in the early molasses experiments were consistent with the so-called Doppler limit, which amounts to a few hundred microkelvin in most alkalis. Light was first used to hold (trap) atoms using the dipole force exerted by a strongly focused laser beam (Chu et al., 1986). In 1987 and 1988 there were two major advances that became central features of the method of creating BEC. First, a practical spontaneous-force trap, the magneto-optical trap (MOT) was demonstrated (Raab et al., 1987); and second, it was observed that under certain conditions, the temperatures in optical molasses are in fact much colder than the Doppler limit (Lett et al., 1988; Chu et al., 1989; Dalibard et al., 1989). The MOT had the essential elements needed for a widely useful optical trap: it required relatively modest amounts of laser power, it was much deeper than dipole traps, and it could capture and hold relatively large numbers of atoms. These were heady times in the laser-cooling business. With experiment yielding temperatures mysteriously far below what theory would predict, it was clear that we all lived under the authority of a munificent God.

During the mid 1980s one of us (Carl) began investigating how useful the technology of laser trapping and cooling could become for general use in atomic physics. Originally this took the form of just making it cheaper and simpler by replacing the expensive dye lasers with 
vastly cheaper semiconductor lasers, and then searching for ways to allow atom trapping with these low-cost but also low-power lasers (Pritchard et al., 1986; Watts and Wieman, 1986). With the demonstration of the MOT and sub-Doppler molasses Carl's group began eagerly studying what physics was limiting the coldness and denseness of these trapped atoms, with the hope of extending the limits further. They discovered that several atomic processes were responsible for these limits. Light-assisted collisions were found to be the major loss process from the MOT as the density increased (Sesko et al., 1989). However, even before that became a serious problem, the light pressure from reradiated photons limited the density (Walker et al., 1990; Sesko et al., 1991). At about the same time, the sub-Doppler temperatures of molasses found by Phillips, Chu, and Cohen-Tannoudji were shown to be due to a combination of light-shifts and optical pumping that became known as Sysiphus cooling (Dalibard and CohenTannoudji, 1989). Random momentum fluctuations from the scattered photons limit the ultimate temperature to about a factor of 10 above the recoil limit. In larger samples, the minimum temperature was higher yet, because of the multiple scattering of the photons. While carrying out studies on the density limits of MOT's Carl's group also continued the effort in technology development. This resulted in the creation of a useful MOT in a simple glass vapor cell (Monroe et al., 1990), thereby eliminating the substantial vacuum chamber required for the slowed atomic beam loading that had previously been used.

Seeking to take advantage of the large gains in phasespace density provided by the MOT while avoiding the limitations imposed by the undesirable effects of photons, Carl and his student Chris Monroe decided to try loading the cold MOT atoms into a magnetic trap (Monroe et al., 1990; see Fig. 2). This worked remarkably well. Because further cooling could be carried out as the atoms were transferred between optical and magnetic trap it was possible to get very cold samples, the coldest that had been produced at that time. More importantly, these were not optical molasses samples that were quickly disappearing but rather magnetically trapped samples that could be held and studied for extended periods. These samples were about a hundred times colder than any previous trapped atom samples, with a correspondingly increased phase-space density. This was a satisfying achievement, but as much as the result itself, it was the relative simplicity of the apparatus required that inspired us (including now Eric Cornell, who joined the project as a postdoc in 1990) to see just how far we could push this marriage of laser cooling and trapping and magnetic trapping.

Previous laser traps involved expensive massive laser systems and large vacuum chambers for atomic beam precooling. Previous magnetic traps for atoms were usually (Bagnato et al., 1987; Doyle, 1991) extremely complex and bulky (often with superconducting coils) because of the need to have sufficiently large depths and strong confinement. Laser traps and magnetic traps were both somewhat heroic experiments individually, to be undertaken only by a select handful of well-equipped AMO laboratories. The prospect of trying to get both traps working, and working well, in the same room and on the same day, was daunting. However, in the first JILA magnetic trap experiment our laser sources were simple diode lasers, the vacuum system was a small glass vapor cell, and the magnetic trap was just a few turns of wire wrapped around it. This magnetic field was adequate because of the low temperatures of the lasercooled and trapped samples. Being able to produce such cold and trapped samples in this manner encouraged one to fantasize wildly about possible things to do with such an atom sample. Inspired by the spin-polarized hydrogen work, our fantasizing quickly turned to the idea of evaporative cooling further to reach BEC. It would require us to increase the phase-space density by 5 orders of magnitude, but since we had just gained about 15 orders of magnitude almost for free with the vapor cell MOT, this did not seem so daunting.

The JILA vapor-cell MOT (Fig. 3), with its superimposed ion pump trap, introduced a number of ideas that are now in common use in the hybrid trapping business (Monroe et al., 1990; Monroe, 1992): (i) Vapor-cell (rather than beam) loading, (ii) fused-glass rather than welded-steel architecture, (iii) extensive use of diode lasers, (iv) magnetic coils located outside the chamber, (v) overall chamber volume measured in cubic centimeters rather than liters, (vi) temperatures measured by imaging an expanded cloud, (vii) magnetic-field curvatures calibrated in situ by observing the frequency of dipole and quadrupole (sloshing and pulsing) cloud motion, (viii) the basic approach of a MOT and a magnetic trap which are spatially superimposed (indeed, which often share some magnetic coils) but temporally sequential, and (ix) optional use of additional molasses and optical pumping sequences inserted in time between the MOT and magnetic trapping stages. It is instructive to note how a modern, Ioffe-Pritchard-based BEC device (Fig. 4) resembles its ancestor (Fig. 3).

As we began to think about applying the technique of evaporative cooling with hydrogen to our very cold alkali atoms we looked carefully at the hydrogen work and its lessons. When viewed from our 1990 perspective the previous decade of work on polarized hydrogen provided a number of important insights. It was clear that the unique absence of any bound states for spinpolarized hydrogen was actually not an important issue (other than its being the catalyst for starting the entire field, of course!). Bound states or not, a very cold sample of spin-polarized hydrogen, like every other gas, has a lower-energy state to which it can go, and its survival depends on the preservation of metastability. For hydrogen the lower-energy state is a solid, although from an experimental point of view the rate-limiting process is the formation of diatomic molecules (with appropriately reoriented spins). Given that all atomic gases are only metastable at the BEC transition point, the real experimental issue becomes: How well can one 


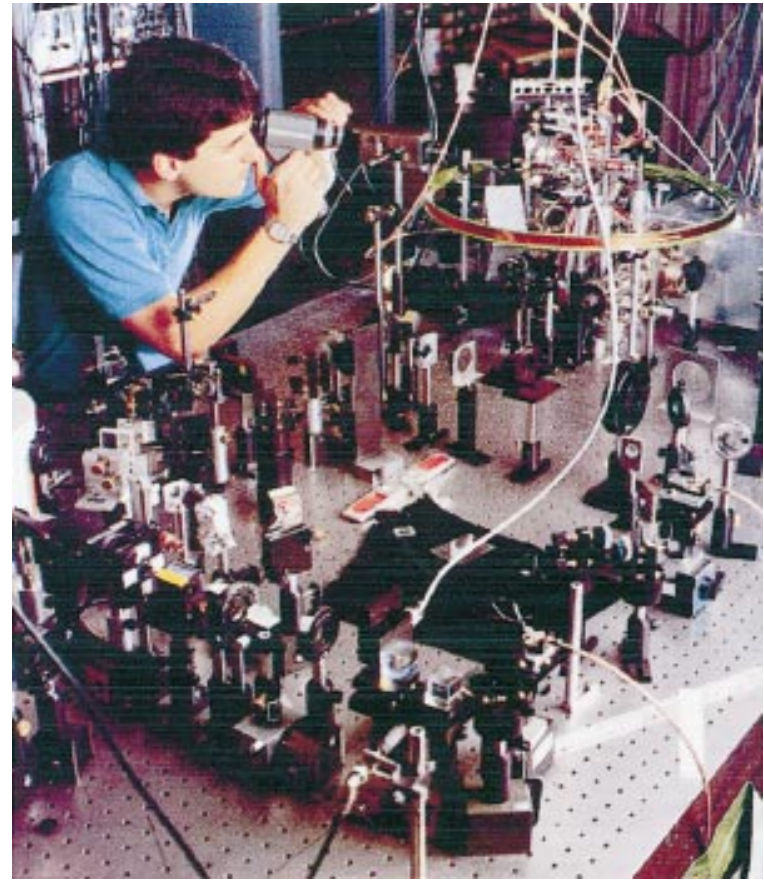

FIG. 2. Chris Monroe examines an early hybrid MOTmagnetic trap apparatus [Color].

preserve the requisite metastability while still cooling sufficiently far to reach BEC?

The realization that metastability was the key experimental challenge one should focus on was probably at least as important to the attainment of BEC as any of the experimental techniques we subsequently developed to actually achieve it. The work on hydrogen provided an essential guide for evaluating and tackling this challenge. It provided us with a potential cooling technique (evaporative cooling of magnetically trapped atoms) and mapped out many of the processes by which a magnetically trapped atom can be lost from its metastable state.

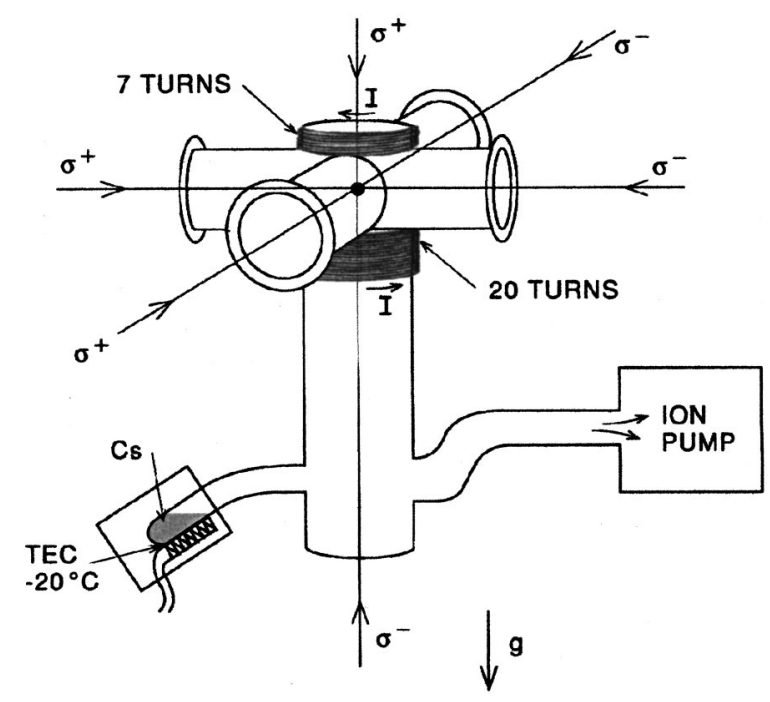

FIG. 3. The glass vapor cell and magnetic coils used in early JILA efforts to hybridize laser cooling and magnetic trapping (see Monroe et al., 1990). The glass tubing is $2.5 \mathrm{~cm}$ in diameter. The Ioffe current bars have been omitted for clarity.

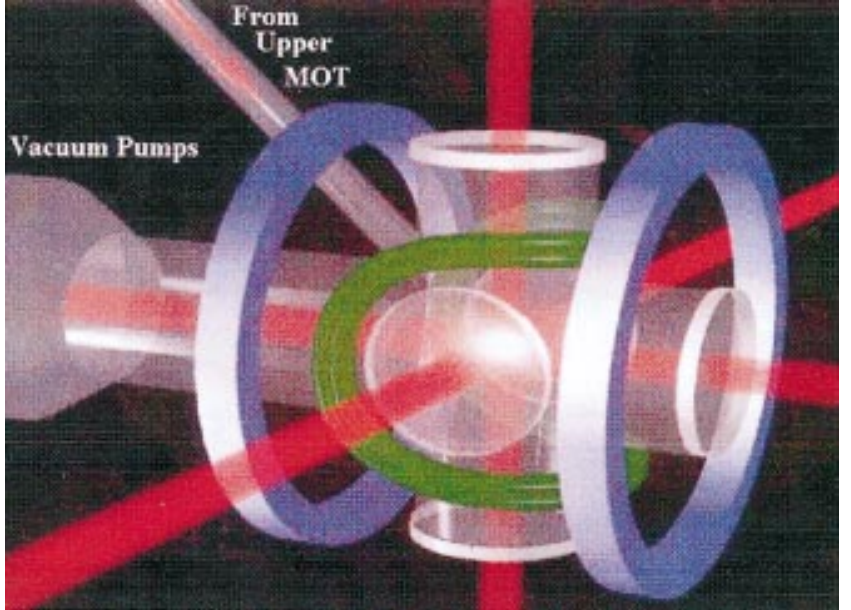

FIG. 4. Modern MOT and magnetic trap apparatus, used by Cornish et al., 2000 [Color].

The hydrogen work made it clear that it was all an issue of good versus bad collisions. The good collisions are elastic collisions that rethermalize the atoms during evaporation. The more collisions there are, the more quickly and efficiently one can cool. The bad collisions are the inelastic collisions that quench the metastability. Hydrogen had already shown that three-body recombination collisions and dipole spin-flip collisions were the major inelastic culprits. The fact that hydrogen researchers were fairly close to reaching BEC was also a strong encouragement. It meant that the goal was not ridiculously distant and that one only had to do a little better in the proportion of good to bad collisions to succeed.

The more we thought about this, the more we began to suspect that our heavy alkali atoms would likely have more favorable collision properties than hydrogen atoms and thus have a good chance of success. Although knowledge of the relevant collision cross sections was totally nonexistent at that time, we were able to come up with arguments for how the cross sections might scale relative to hydrogen. These are discussed in more detail below in the section discussing why collisional concerns make it likely that BEC can be created in a large number of different species. Here we will just give a brief summary consistent with our views circa 1990. The dipole spin-flip collisions that limited hydrogen involve spin-spin interactions and thus could be expected to be similar for the alkalis and for hydrogen because the magnetic moments are all about the same. The good collisions needed for evaporative cooling, however, should be much larger for heavy alkalis with their fat fluffy electron clouds than for hydrogen. The other villain of the hydrogen effort, three-body recombination, was a total mystery, but because it goes as density cubed while the good elastic collisions go as density squared, it seemed as if we should always be able to find a sufficiently low-density and low-temperature regime to avoid it (see Monroe, 1992).

As a minor historical note, we might point out that during these considerations we happily ignored the fact that the temperatures required to achieve $\mathrm{BEC}$ in a 
heavy alkali gas are far colder than those needed for the same density of hydrogen. The critical temperature for ideal-gas BEC is inversely proportional to the mass. It was clear that we would need to cool to well under a microkelvin, and a large three-body recombination rate would have required us to go to possibly far lower temperatures. To someone coming from a traditional cryogenics background this would (and probably did) seem like sheer folly. The hydrogen work had been pushing hard for some years at the state of the art in cryogenic technology, and here we proposed to happily jump far beyond that. Fortunately we were coming to this from an AMO background in a time when temperatures achieved by laser cooling were dropping through the floor. Optimism was in the air. In fact, we later discovered optimism can take one only so far: There were actually considerable experimental difficulties, and further cooling came at some considerable effort and a five-year delay. Nevertheless, it is remarkable that with evaporative cooling a magnetically trapped sample of atoms, surrounded on all sides by a 300-K glass cell, can be cooled to reach temperatures of only a few nanokelvin, and moreover it looks quite feasible to reach even colder temperatures.

General collisional considerations gave us some hope that the evaporative cooling hybrid trap approach with alkali atoms would get us to BEC, or, if not, at least reveal some interesting new physics that would prevent it. Nonetheless, there were powerful arguments against pursuing this. First, our 1990-era arguments in favor of it were based on some very fuzzy intuition; there were no collision data or theories to back it up and there were strong voices in disagreement. Second, the hydrogen experiments seemed to be on the verge of reaching BEC, and in fact we thought it was likely that if BEC could be achieved they would succeed first. However, our belief in the virtues of our technology really carried the day in convincing us to proceed. With convenient lasers in the near-IR, and with the good optical access of a roomtemperature glass cell, detection sensitivity could approach single-atom capability. We could take pictures of only a few thousand trapped atoms and immediately know the energy and density distribution. If we wanted to modify our magnetic trap it only required a few hours winding and installing a new coil of wires. This was a dramatic contrast with the hydrogen experiments that, like all state-of-the-art cryogenics experiments, required an apparatus that was the better part of two stories, and the time to modify it was measured in (large) fractions of a year. Also, atomic hydrogen was much more difficult to detect and so the diagnostics were far more limited. This convinced us that although hydrogen would likely succeed first, our hybrid trap approach with easily observed and manipulated alkali samples would be able to carry out important science and so was well worth pursuing in its own right.

From the very beginning in 1990, our work on BEC was heavily involved with cold atomic collisions. This was somewhat ironic since previously both of us had actively avoided the large fraction of AMO work on the subject of atomic collisions. Atomic collisions at very cold temperatures is now a major branch of the discipline of AMO physics, but at the end of the 1980s there were almost no experimental data, and what there was came in fact from the spin-polarized hydrogen experiments (Gillaspy et al., 1989). There was theoretical work on hydrogen from Shlyapnikov and Kagan (Kagan et al., 1981, 1984), and from Silvera and Verhaar (Lagendijk et al., 1986). An early paper by Pritchard (1986) includes estimates on low-temperature collisional properties for alkalis. His estimates were extrapolations from roomtemperature results, but in retrospect, several were surprisingly accurate. As we began to work on evaporative cooling, much of our effort was devoted to determining the sizes of all the relevant good and bad collision cross sections. Our efforts were helped by the theoretical efforts of Boudewijn Verhaar, who was among the first to take our efforts seriously and attempt to calculate the rates in question. Chris Greene also provided us with some useful theoretical estimates.

Starting in 1990 we carried out a series of experiments exploring various magnetic traps and measuring the relevant collision cross sections. As this work proceeded we developed a far better understanding of the conditions necessary for evaporative cooling and a much clearer understanding of the relevant collisional issues (Monroe et al., 1993; Newbury et al., 1995). Our experimental concerns evolved accordingly. In the early experiments (Monroe et al., 1990, 1993; Cornell et al., 1991; Monroe, 1992) a number of issues came up that continue to confront all BEC experiments: the importance of aligning the centers of the MOT and the magnetic trap, the density-reducing effects of mode-mismatch, the need to account carefully for the (previously ignored) force of gravity, heating (and not merely loss) from background gas collisions, the usefulness of being able to turn off the magnetic fields rapidly, the need to synchronize many changes in laser status and magnetic fields together with image acquisition, an appreciation for the many issues that can interfere with accurate determinations of density and temperature by optical methods, either florescence or absorption imaging, and careful stabilization of magnetic fields. The mastery of these issues in these early days made it possible for us to proceed relatively quickly to quantitative measurements with the BEC once we had it.

In 1992 we came to realize that dipolar relaxation in alkalis should in principle not be a limiting factor. As explained in the final section of this article, collisional scaling with temperature and magnetic field is such that, except in pathological situations, the problem of good and bad collisions in the evaporative cooling of alkalis is reduced to the ratio of the elastic collision rate to the rate of loss due to imperfect vacuum; dipolar relaxation and three-body recombination can be finessed, particularly since our preliminary data showed they were not enormous. It was reassuring to move ahead on efforts to evaporate with the knowledge that, while we were essentially proceeding in the dark, there were not as many monsters in the dark as we had originally imagined. 
It rapidly became clear that the primary concerns would be having sufficient elastic collision rate in the magnetic trap and sufficiently low background pressure to have few background collisions that removed atoms from the trap. To accomplish this it was clear that we needed higher densities in the magnetic trap than we were getting from the MOT. Our first effort to increase the density two years earlier was based on a multipleloading scheme (Cornell et al., 1991). Multiple MOTloads of atoms were launched in moving molasses, optically pumped into an untrapped Zeeman level, focused into a magnetic trap, then optically repumped into a trapped level. The repumping represented the necessary dissipation, so that multiple loads of atoms could be inserted in a continuously operating magnetic trap. In practice, each step of the process involved some losses, and the final result was disappointing. Later, however, as discussed below, we resurrected the idea of multiple loading from one MOT to another to good advantage (Gibble et al., 1995; Myatt et al., 1996). This is now a technique currently in widespread practice.

In addition to building up the initial density we realized that the collision rate could be dramatically increased by, after loading into a magnetic trap, compressing the atoms by further increasing the curvature of the confining magnetic fields. In a harmonic trap, the collision rate after adiabatic compression scales as the final confining frequency squared (Monroe, 1992). This method is discussed by Monroe (1992) and was implemented first in early ground-state collisional work (Monroe et al., 1993).

In fall of 1992, Eric's postdoctoral appointment concluded, and, after a tour through the job market, he decided to take the equivalent of an assistant professor position at JILA/NIST. He decided to use his startup money to build a new experimental apparatus that would be designed to put these ideas together to make sure evaporation worked as we expected. Meanwhile, we continued to pursue the possibility of enhanced collision cross sections in cesium using a Feshbach resonance. At that point our Monte Carlo simulations said that a ratio of about 150 elastic collisions per trap lifetime was required to achieve runaway evaporation. This is the condition where the elastic collision rate would continue to increase as the temperature decreased, and hence evaporation would continue to improve as the temperature was reduced. We also had reasonable determinations of the elastic collision cross sections.

So the plan was to build a simple quadrupole trap that would allow very strong squeezing to greatly enhance the collision rate, combined with a good vacuum system in order to make sure evaporative cooling worked as expected. Clearly, there was much to be gained by building a more tightly confining magnetic trap, but the requirement of adequate optical access for the MOT, along with engineering constraints on power dissipation, made the design problem complicated.

When constructing a trap for weak-field-seeking atoms, with the aim of confining the atoms to a spatial size much smaller than the size of the magnets, one would like to use linear gradients. In that case, however, one is confronted with the problem of the minimum in the magnitude of the magnetic fields (and thus of the confining potential) occurring at a local zero in the magnetic field. This zero represents a hole in the trap, a site at which atoms can undergo Majorana transitions (Majorana, 1931) and thus escape from the trap. If one uses the second-order gradients from the magnets to provide the confinement, there is a marked loss of confinement strength. This scaling is discussed by Petrich et al. (1995). We knew that once the atoms became cold enough they would leak out the hole in the bottom of the trap, but the plan was to go ahead and get evaporation and worry about the hole later. We also recognized that even with successful evaporative cooling, and presuming we could solve the issue of the hole in the quadrupole trap, there was still the question of the sign of scattering length, which must be positive to ensure the stability of a large condensate.

In setting up the new apparatus Eric chose to use rubidium. Given the modulo arithmetic that goes into determining a scattering length, it seemed fair to treat the scattering lengths of different isotopes as statistically independent events, and rubidium with its two stable isotopes offered two rolls of the dice for the same laser system. Eric then purchased a set of diode lasers for the rubidium wavelength, but of course we kept the original cesium-tuned diode lasers. The wavelengths of cesium and of the two rubidium isotopes are sufficiently similar that in most cases one can use the same optics. Thus we preserved the option of converting from one species to another in a matter of weeks. The chances then of Nature's conspiring to make the scattering length negative, for both hyperfine levels, for all three atoms, seemed very small.

Progress in cold collisions, particularly the experiment and theory of photoassociative collisions, had moved forward so rapidly that by the time we had evaporatively cooled rubidium to close to BEC temperatures a couple of years later there existed, at the $20 \%$ level, values for several of the elastic scattering lengths. In particular, we knew that it was positive for the 2,2 state of $\mathrm{Rb}-87$ (Thorsheim et al., 1987; Lett et al., 1993; Miller et al., 1993; Abraham et al., 1995; Gardner et al., 1995; McAlexander et al., 1995).

Our original idea for the quadrupole trap experiment was to pulse a burst of rubidium into our cell, where we would catch a large sample in the MOT and then hold it as the residual rubidium was quickly pumped away, leaving a long trap lifetime. We, particularly Eric's postdoc, Mike Anderson, spent many frustrating months discovering how difficult this seemingly simple idea was to actually implement in practice. The manner in which rubidium interacted with glass and stainless-steel surfaces conspired to make this so difficult we finally gave up. We ended up going with a far-from-optimum situation of working with extremely low rubidium pressure and doing our best at maximizing the number of atoms captured in the MOT from this feeble vapor and enhancing the collision rate for those relatively few atoms as much 
as possible. We recognized that this was a major compromise, but we had been trying to evaporate for some time, and we were getting impatient! We had no stomach for building another apparatus just to see evaporation. Fortunately we were able to find two key elements to enhance the MOT loading and density. First was the use of a dark-spot MOT in which there is a hole in the center of the MOT beams so the atoms are not excited. This technique had been demonstrated by Ketterle (Ketterle et al., 1993) as a way to greatly enhance the density of atoms in a MOT under conditions of a very high loading rate. The number of atoms we could load in our vapor cell MOT with very low rubidium vapor was determined by the loading rate over the loss rate. In this case the loss rate was the photoassociative collisions we had long before found to be important for losses from MOT's. The dark-spot geometry reduced this two-body photoassociative loss in part because in our conditions it reduced the density of atoms in the MOT (Anderson et al., 1994).

Using this approach we were able to obtain $10^{8}$ atoms in the MOT collected out of a very low vapor background (so that magnetic trap lifetime was greater than $100 \mathrm{~s}$ ). The second key element was the invention of the compressed MOT (CMOT), a technique for substantially enhancing the density of atoms in the MOT on a transient basis. For the CMOT, the MOT was filled and then the field gradient and laser detuning were suddenly changed to greatly suppress the multiple photon scattering. This produced much higher densities and clouds whose shape was a much better match to the desired shape of the cloud in the magnetic trap. This was a very transient effect because the losses from the MOT were much larger under these conditions, but that was not important; the atoms needed only to be held for the milliseconds required before they were transferred to the magnetic trap (Petrich et al., 1994; see Fig. 5). With these improvements and a quadrupole trap that provided substantial squeezing, we were able to finally demonstrate evaporative cooling in rubidium.

Cooling by evaporation is a process found throughout Nature. Whether the material being cooled is an atomic nucleus or the Atlantic Ocean, the rate of natural evaporation and the minimum temperature achievable are limited by the particular fixed value of the work function of the evaporating substance. In magnetically confined atoms, no such limit exists, because the work function is simply the height of the lowest point in the rim of the confining potential. Hess (1986) pointed out that, by perturbing the confining magnetic fields, one could make the work function of a trap arbitrarily low; as long as favorable collisional conditions persist, there is no lower limit to the temperatures attainable in this forced evaporation.

Pritchard (Pritchard et al., 1989) pointed out that evaporation could be performed more conveniently if the rim of the trap were defined by an rf-resonance condition, rather than simply by the topography of the magnetic field; experimentally, his group made first use of position-dependent $\mathrm{rf}$ transitions to selectively transfer magnetically trapped sodium atoms between Zeeman levels and thus characterized their temperature (Martin et al., 1988). In our experiment we used Pritchard's technique of an rf field to selectively evaporate.

It was a great relief to see evaporative cooling of laser precooled, magnetically trapped atoms finally work, as we had been anticipating it would for so many years. Unfortunately, it worked exactly as well, but no better, than we had anticipated. The atoms were cooled to about $40 \mu \mathrm{K}$ and then disappeared, at just the temperature we had estimated they would be lost, through the hole in the bottom of the quadrupole trap. Eric came up with an idea that solved this problem. It was a design for a new type of trap that required relatively little modification to the apparatus and so was quickly implemented. This was the Time Orbiting Potential (TOP) trap in which a small rotating magnetic field was added to the quadrupole field (Petrich et al., 1995). This moved the field zero in an orbit faster than the atoms could follow. It was the perfect solution to our problem.

Mike Anderson, another postdoc, Wolfgang Petrich, and graduate student Jason Ensher quickly implemented this design. Their efforts were spurred on by the realization that there were several other groups who had now demonstrated or were known to be on the verge of demonstrating evaporative cooling in alkalis in the pursuit of BEC. The TOP design worked well, and the samples were cooled far colder, in fact too cold for us to reliably measure. We had been measuring temperature simply by looking at the spatial size of the cloud in the magnetic trap. As the temperature was reduced the size decreased, but we were now reaching temperatures so low that the size had reached the resolution limit of the optical system. We saw dramatic changes in the shapes of the images as the clouds became very small, but we knew that a variety of diffraction and aberration effects could greatly distort images when the sample size became only a few wavelengths in size, so our reaction to these shapes was muted, and we knew we had to have better diagnostics before we could have meaningful results. Here we were helped by our long experience in studying various trapped clouds over the years. We already knew the value of turning the magnetic trap off to let the cloud expand and then imaging the expanded cloud to get a measure of the momentum distribution in the trap. Since the trap was harmonic, the momentum distribution and the original density distribution were nearly interchangeable. Unfortunately, once the magnetic field was off, the atoms not only expanded but also simply fell under the influence of gravity. We found that the atoms tended to fall out of the field of view of our microscope before they had sufficiently expanded. The final addition to the apparatus was a supplementary magnetic coil, which provided sufficient field gradient to cancel the effects of gravity while minimizing any perturbation to the relative ballistic trajectories of the expanding atoms.

Anderson, Ensher, and a new graduate student, Mike Matthews (Fig. 6), worked through a weekend to install the antigravity coil and, after an additional day or two of 


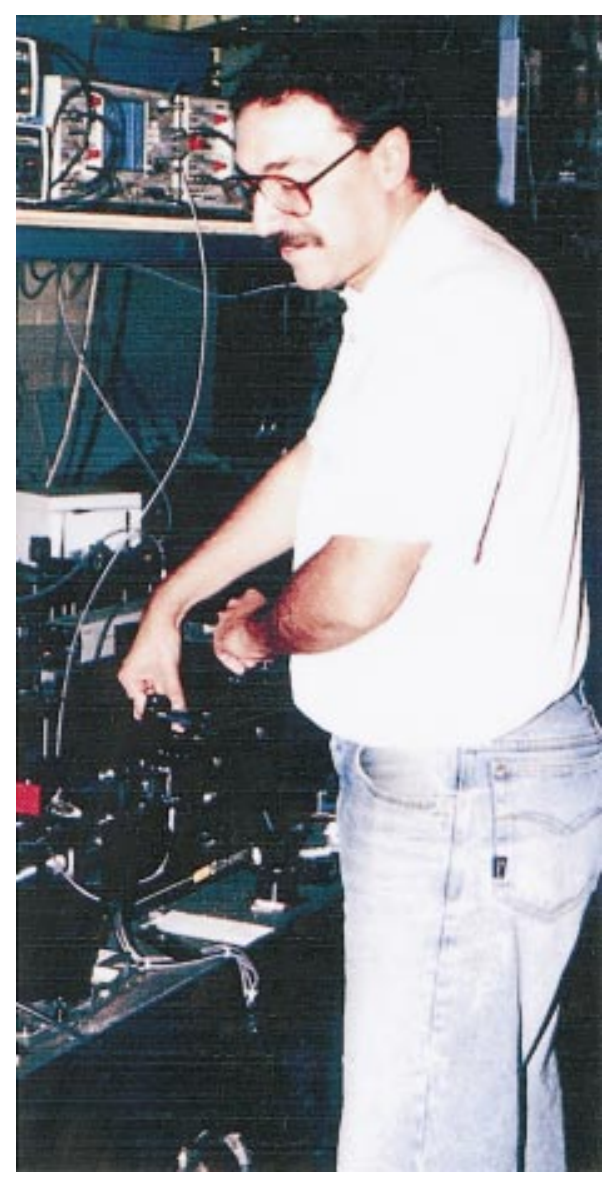

FIG. 5. Wolfgang Petrich working on CMOT [Color].

trial and error, got the new field configuration shimmed up. By June 5, 1995 the new technology was working well and we began to look at the now greatly expanded clouds. To our delight, the long-awaited two-component distribution was almost immediately apparent (Fig. 7) when the samples were cooled to the regime where BEC was expected. The excitement was tempered by the con-

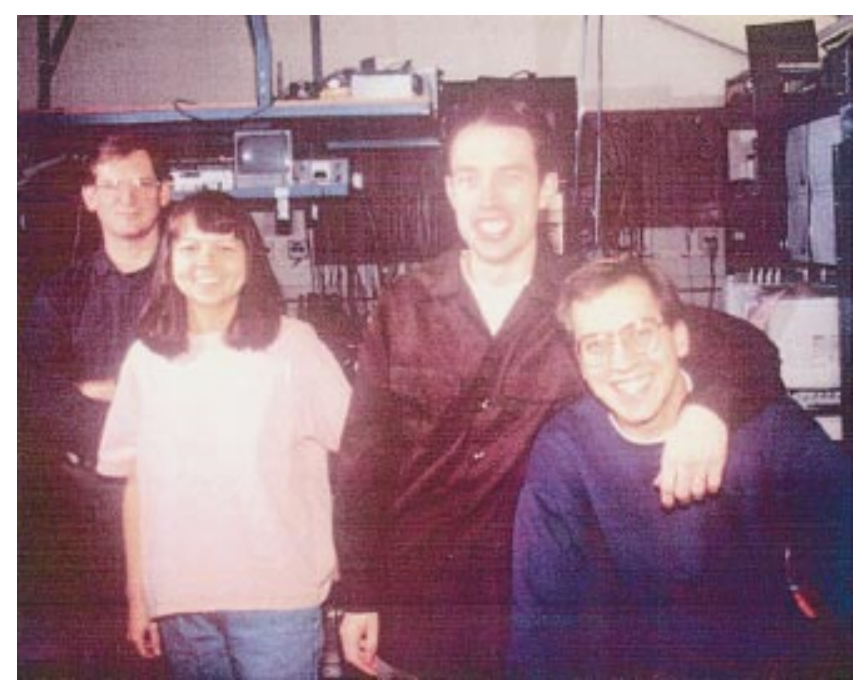

FIG. 6. From left, Mike Anderson, Debbie Jin, Mike Matthews, and Jason Ensher savor results of early BEC experiment [Color]. cern that after so many years of anticipating two component clouds as a signature of BEC, we might be fooling ourselves.

Almost from the beginning of the search for BEC, it was recognized (Lovelace and Tommila, 1987) that as the sample started to condense, there would be a spike in the density and momentum distributions corresponding to the macroscopic population of the ground state. This would show up as a second component on top of the much broader normal thermal distribution of uncondensed atoms. This was the signature we had been hoping to see from our first days of contemplating BEC. The size of the BEC component in these first observations also seemed almost too good to be true. In those days it was known that in the much higher density of the condensate, three-body recombination would be a more dominant effect than in the lower-density uncondensed gas. For hydrogen it was calculated that the condensed component could never be more than a few percent of the sample. The three-body rate constants were totally unknown for alkali atoms at that time, but because of the $\mathrm{H}$ results it still seemed reasonable to expect the condensate component might only be a modest fraction of the total sample. But in our first samples we saw it could be nearly $100 \%$ ! In the light of the prevailing myth of unattainability that had grown up around BEC over the years, our observations seemed too good to be true. We were experienced enough to know that when results in experimental physics seem too good to be true, they almost always are! We worried that in our enthusiasm we might confuse the long-desired BEC with some spurious artifact of our imaging system.

However, our worries about the possibility of deluding ourselves were quickly and almost entirely alleviated by the anisotropy of the BEC cloud. This was a very distinctive signature of BEC, the credibility of which was greatly enhanced to us by the fact that it first revealed itself in the experiment, and then we recognized its significance, rather than vice versa. It was a somewhat fortuitous accident that the TOP trap provided a distinctly anisotropic trapping potential, since we did not appreciate its benefits until we saw the BEC data. A normal thermal gas (in the collisionally thin limit) released from an anisotropic potential will spread out isotropically. This is required by the equipartition theorem. However, a Bose-Einstein condensate is a quantum wave and so its expansion is governed by a wave equation. The more tightly confined direction will expand the most rapidly, a manifestation of the uncertainty principle. Seeing the BEC component of our two-component distribution display just this anisotropy, while the broader uncondensed portion of the sample observed at the same time, with the same imaging system, remained perfectly isotropic (as shown in Fig. 8), provided the crucial piece of corroborating evidence that this was the long-awaited BEC.

By coincidence we were scheduled to present progress reports on our efforts to achieve BEC at three international conferences in the few weeks following these observations (Anderson et al., 1996). Nearly all the experts in the field were represented at one or more of these 


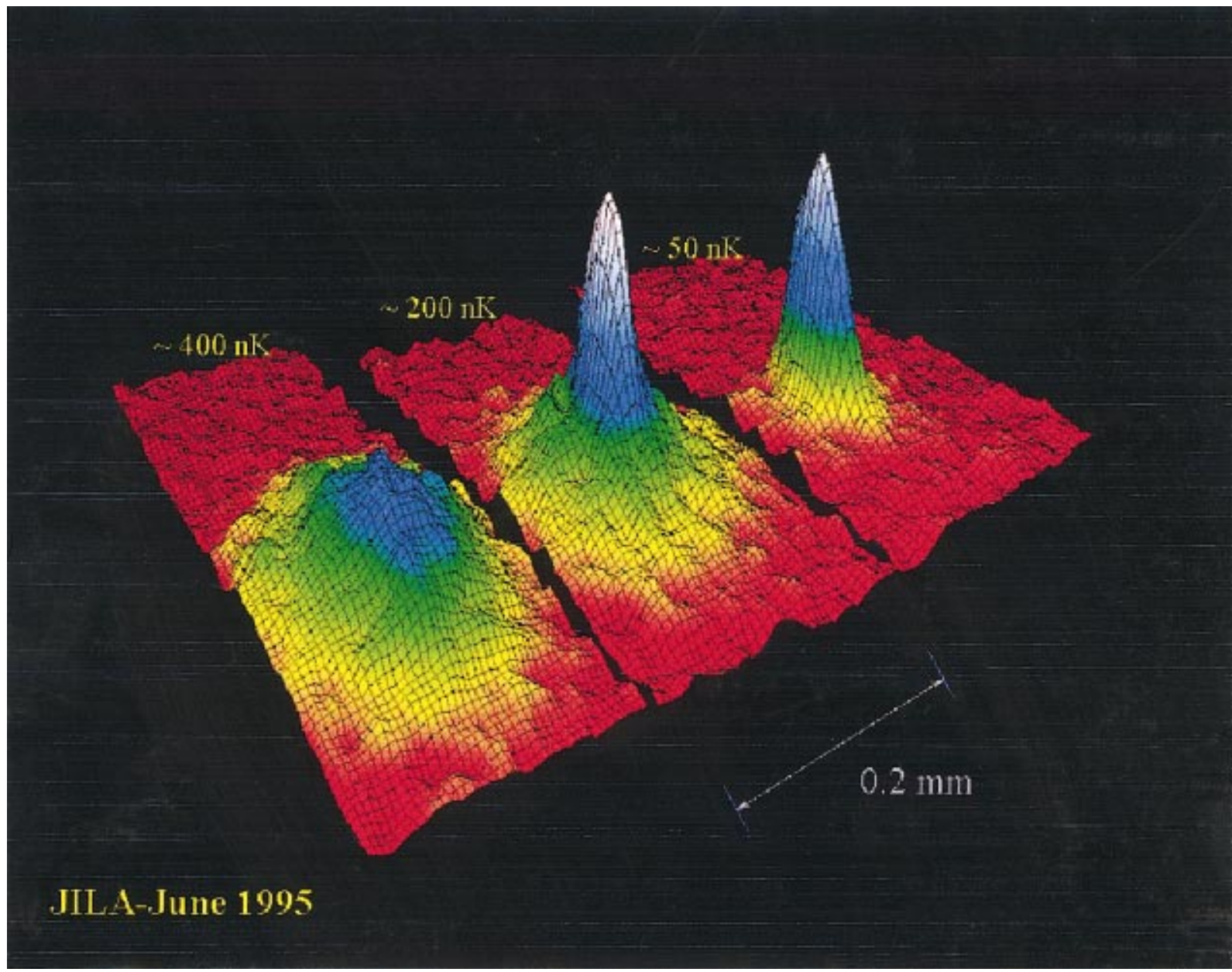

FIG. 7. Three density distributions of the expanded clouds of rubidium atoms at three different temperatures. The appearance of the condensate is apparent as the narrow feature in the middle image. On the far right, nearly all the atoms in the sample are in the condensate. The original experimental data were two-dimensional black and white shadow images, but these images have been converted to three dimensions and given false color density contours [Color].

conferences, and the data were sufficient to convince the most skeptical of them that we had truly observed BEC. This consensus probably facilitated the rapid refereeing and publication of our results.
In the original TOP-trap apparatus we were able to obtain so-called pure condensates of a few thousand atoms. By pure condensates we meant that nearly all the atoms were in the condensed fraction of the sample.
A

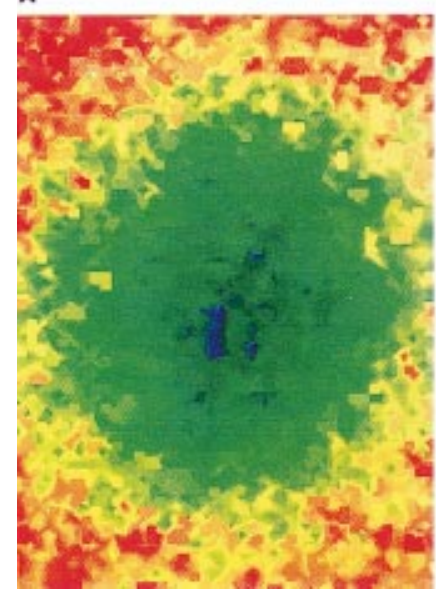

B

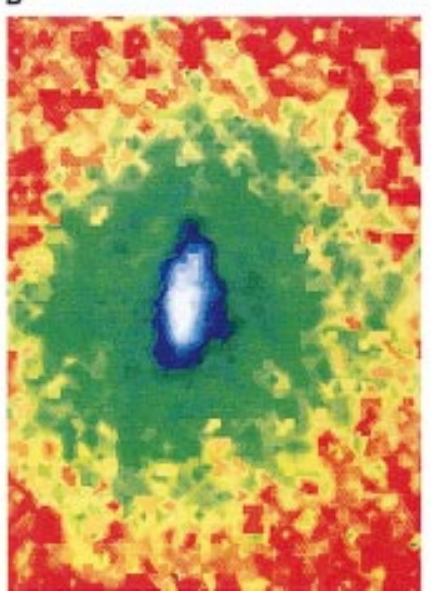

C

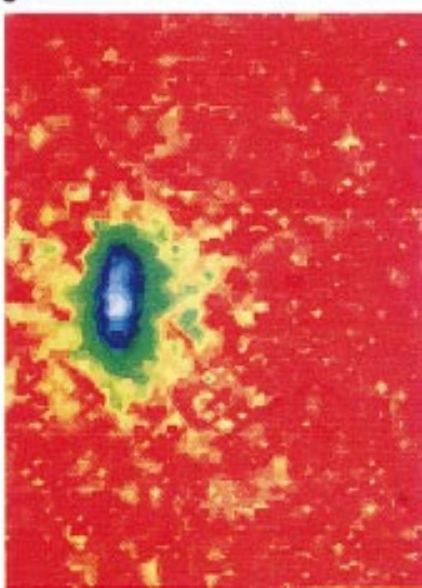

FIG. 8. Looking down on the three images of Figure 7 (Anderson et al., 1995). The condensate in B and C is clearly elliptical in shape [Color]. 
Samples of this size were easily large enough to image. Over the few months immediately following the original observation, we undertook the process of a technological shoring up of the machine, until the machine reached the level of reliability necessary to crank out condensate after reproducible condensate. This set the stage for the first generation of experiments characterizing the properties of the condensate, most notably the condensate excitation studies discussed below.

Although by 1995 and 1996 we were able to carry out a number of significant BEC experiments with the original TOP-trap machine, even by 1994, well before the original condensates were observed, we had come to realize the limitations of the single-cell design. Our efforts to modulate the vapor pressure were not very successful, which forced us to operate at a steady-state rubidium vapor pressure. Choosing the value of vapor pressure at which to operate represented a compromise between our need to fill the vapor-cell MOT with as many atoms as possible and our need to have the lifetime in the magnetic trap as long as possible. The single-cell design also compelled us to make a second compromise, this time over the size of the glass cell. The laser beams of the MOT enter the cell through the smooth, flat region of the cell; the larger the glass cell, the larger the MOT beams, and the more atoms we could herd into the MOT from the room-temperature background vapor. On the other hand, the smaller the glass cell, the smaller the radii of the magnetic coils wound round the outside of the cell, and the stronger the confinement provided by the magnetic trap. Hans Rohner in the JILA specialty shop had learned how (Rohner, 1994) to create glass cells with the minimum possible wasted area. But even with the dead space between the inner diameter of the magnetic coils and the outer diameter of the clear glass windows made as small as it could be, we were confronted with an unwelcome tradeoff.

Thus, in 1994, in parallel with our efforts to push as hard as we could toward BEC in our original, single-cell TOP trap, we began working on a new technology that would avoid this painful tradeoff. This approach was a modified version of our old multiple loading scheme in which many loads from a MOT were transferred to a magnetic trap in a differentially pumped vacuum chamber. That approach had been defeated by the difficulty in transferring atoms from MOT to magnetic trap without losing phase-space density. There was no dissipation in the magnetic trap to compensate for a slightly too hard or too soft push from one trap to the other. This made us recognize the importance of having dissipation in the second trap, and so we went to a system in which atoms were captured in a large-cell MOT in a region of high rubidium pressure, and then transferred through a small tube into a second, small-cell MOT in a low-pressure region. This eliminated the previous disadvantages while preserving the advantages of multiple loading to get much larger numbers of trapped atoms in a low-vacuum region. The approach worked well, particularly when we found that simple strips of plastic refrigerator magnet material around the outside of the transfer tube between the two traps provided an excellent guide to confine the atoms as they were pushed from one trap to the other (Myatt et al., 1996).

With this scheme we were still able to use inexpensive low-power diode lasers to obtain about one hundred times more atoms in the magnetic trap than in our single MOT-loaded TOP magnetic trap and with a far longer lifetime; we saw trap lifetimes up to $1000 \mathrm{~s}$ in the double MOT magnetic trap. This system started working in 1996 and it marked a profound difference in the ease with which we could make BEC (Myatt et al., 1997). In the original BEC experiment everything had to be very well optimized to achieve the conditions necessary for runaway evaporative cooling and thereby BEC. In the double MOT system there were orders of magnitude to spare. Not only did this allow us to routinely obtain million-atom pure condensates, but it also meant that we could dispense with the dark-spot optical configuration with its troublesome alignment. We could be much less precise with many other aspects of the experiment as well.

The first magnetic trap we used with the double-MOT BEC machine was not a TOP trap, but instead was our old baseball-style Ioffe-Pritchard trap. The baseball coil trap is rather complementary to the TOP trap in that each has unique capabilities. For example, the geometry of the TOP trap potential can be changed over a wide range, although the range of dc fields is quite limited. In contrast, the geometry of the baseball coil trap potential can be varied only by small amounts, but the dc bias field can be easily varied over hundreds of gauss. Thus in 1996, when we upgraded the original BEC machine to incorporate the double-MOT technology, we preserved the TOP trap coil design. Each is well suited to certain types of experiments, as will be evident in the discussions below.

With the double-MOT setups we were able to routinely make million-atom condensates in a highly reliable manner in both TOP and baseball-type magnetic traps. These were used to carry out a large number of experiments with condensates over the period from 1996 to the present. Some of our favorite experiments are briefly discussed below.

\section{FAVORITE EXPERIMENTS}

\section{Collective excitations}

In this section, by excitations we mean coherent fluctuations in the density distribution. Excitation experiments in dilute-gas BEC have been motivated by two main considerations. First, a Bose-Einstein condensate is expected to be a superfluid, and a superfluid is defined by its dynamical behavior. Studying excitations is an obvious initial step toward understanding dynamical behavior. Second, in experimental physics a precision measurement is almost always a frequency measurement, and the easiest way to study an effect with precision is to find an observable frequency that is sensitive to that effect. In the case of dilute-gas BEC, the observed fre- 


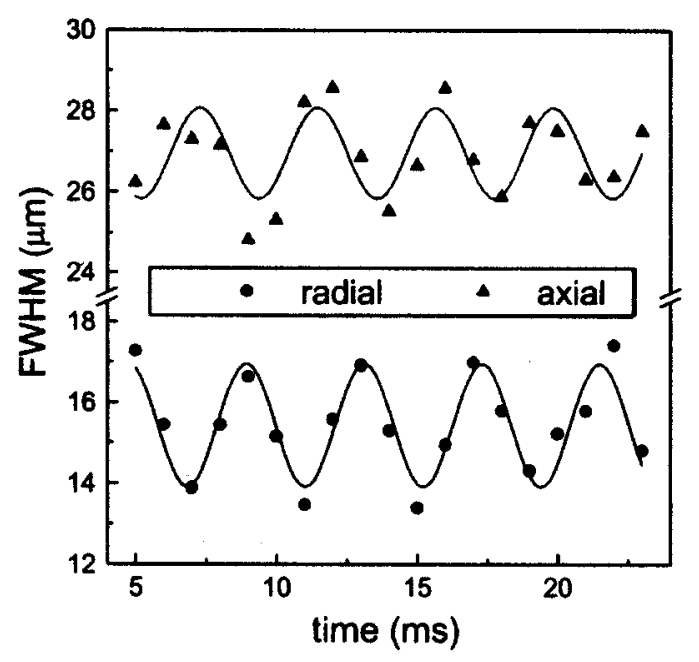

FIG. 9. Zero-temperature excitation data from Jin et al. (1996). A weak $m=0$ modulation of the magnetic trapping potential is applied to a 4500 -atom condensate in a $132-\mathrm{Hz}$ (radial) trap. Afterward, the freely evolving response of the condensate shows radial oscillations. Also observed is a sympathetic response of the axial width, approximately $180^{\circ}$ out of phase. The frequency of the excitation is determined from a sine wave fit to the freely oscillating cloud widths.

quency of standing-wave excitations in a condensate is a precise test of our understanding of the effect of interactions.

BEC excitations were first observed by Jason Ensher, Mike Matthews, and then-postdoc Debbie Jin, using destructive imaging of expanded clouds (Jin et al., 1996). The nearly zero-temperature clouds were coherently excited (see below), then allowed to evolve in the trap for some particular dwell time, and then rapidly expanded and imaged via absorption imaging. By repeating the procedure many times with varying dwell times, the time-evolution of the condensate density profile can be mapped out. From these data, frequencies and damping rates can be extracted. In axially symmetric traps, excitations can be characterized by their projection of angular momentum on the axis. The perturbation on the density distribution caused by the excitation of lowest-lying $m=0$ and $m=2$ modes can be characterized as simple oscillations in the condensate's linear dimensions. Figure 9 shows the widths of an oscillating condensate as a function of dwell time.

A frequency-selective method for driving the excitations is to modulate the trapping potential at the frequency of the excitation to be excited (Jin et al., 1996). Experimentally this is accomplished by summing a small ac component onto the current in the trapping magnets. In a TOP trap, it is convenient enough to independently modulate the three second-order terms in the transverse potential. By controlling the relative phase of these modulations, one can impose $m=0, m=2$, or $m=-2$ symmetry on the excitation drive.

There have been a very large number of theory papers published on excitations; much of this work is reviewed by Dalfovo et al. (1999). All the zero-temperature, small-amplitude excitation experiments published to date have been very successfully modeled theoretically. Quantitative agreement has been by and large very good; small discrepancies can be accounted for by assuming reasonable experimental imperfections with respect to the $T=0$ and small-amplitude requirements of theory.

The excitation measurements discussed above were then revisited at nonzero temperature (Jin et al., 1997). The frequency of the condensate excitations was clearly observed to depend on the temperature, and the damping rates showed a strong temperature dependence. This work is important because it bears on the little-studied finite-temperature physics of interacting condensates. Connection with theory (Hutchinson et al., 1997; Dodd et al., 1998; Fedichev and Shlyapnikov, 1998) remains somewhat tentative. The damping rates, which are observed to be roughly linear in temperature, have been explained in the context of Landau damping (Liu, 1997; Fedichev et al., 1998). The frequency shifts are difficult to understand, in large part because the data so far have been collected in a theoretically awkward, intermediate regime: the cloud of noncondensate atoms is neither so thin as to have completely negligible effect on the condensate, nor so thick as to be deeply in the hydrodynamic (HD) regime. In this context, hydrodynamic regime means that the classical mean free path in the thermal cloud is much shorter than any of its physical dimensions. In the opposite limit, the collisionless regime, there are conceptual difficulties with describing the observed density fluctuations as collective modes. Recent theoretical work suggests that good agreement with experiment may hinge on correctly including the role of the excitation drive (Stoof, 2000; Jackson and Zaremba, 2002).

\section{Two-component condensates}

As mentioned above, the double-MOT system made it possible to produce condensates even if one were quite sloppy with many of the experimental parameters. One such parameter was the spin state in which the atoms are optically pumped before being loaded into the magnetic trap. As our student Chris Myatt was tinkering around setting up the evaporation one day, he noticed, to his surprise, that there seemed to be two different clouds of condensate in the trap. They were roughly at the locations expected for the 2,2 and 1,- 1 spin states to sit, but that seemed impossible to us because these two states could undergo spin-exchange collisions that would cause them to be lost from the trap, and the spinexchange collision cross sections were thought to be enormous. After extensive further studies to try and identify what strange spurious effect must be responsible for the images of two condensate clouds we came to realize that they had to be those two spin states. By a remarkable coincidence, the triplet and singlet phase shifts are identical and so at ultralow temperatures the spin-exchange collisions are suppressed in ${ }^{87} \mathrm{Rb}$ by three to four orders of magnitude! This suppression meant that the different spin species could coexist and their 


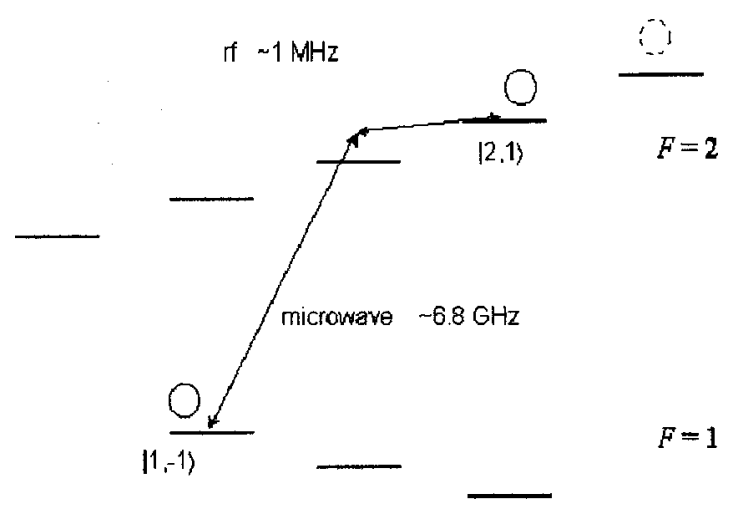

FIG. 10. Energy-level diagram for ground electronic state of ${ }^{87} \mathrm{Rb}$. The first condensates were created in the 2,2 state. Mixtures containing the 2,2 and 1,-1 state were found to coexist. In later studies we created condensates in the $1,-1$ state and then excited it to the 2,1 state using a microwave plus rf twophonon transition.

mixtures could be studied. In early work we showed that one could carry out sympathetic cooling to make BEC by evaporating only one species and using it as a cooling fluid to chill the second spin state (Myatt et al., 1997). We also were able to see how the two condensates interacted and pushed each other apart, excluding all but a small overlap in spite of the fact that they were highly dilute gases.

These early observations stimulated an extensive program of research on two-component condensates. After Myatt's original measurements (Myatt et al., 1997), our work in this field, led by postdoc David Hall, concentrated on the 1,-1 and 2,+1 states (see Fig. 10) because they could be coherently interconverted using twophoton (microwave plus rf) transitions and they had nearly identical magnetic moments and so saw nearly the same trapping potentials (Matthews et al., 1998). When the two-photon radiation field is turned off, the rate of spontaneous interconversion between the two spin species essentially vanishes, and moreover the optical imaging process readily distinguishes one species from the other, as their difference in energy $(6.8 \mathrm{GHz})$ is very large compared to the excited-state linewidth. In this situation, one may model the condensate dynamics as though there were two distinct quantum fluids in the trap. Small differences in scattering length make the two fluids have a marginal tendency to separate spatially, at least in an inhomogeneous potential, but the interspecies healing length is long so that in the equilibrium configuration there is considerable overlap between the two species (Hall et al., 1998a, 1998b). On the other hand, the presence of a near-resonant two-photon coupling drive effectively brings the two energy levels quite close to one another: on resonance, the corresponding dressed energy levels are separated only by the effective Rabi frequency for the two-photon drive. In this limit, one may in a certain sense think of the condensate as being described by a two-level, spinor field (Cornell et al., 1998; Matthews et al., 1999b).

We got a lot of mileage out of this system and continue to explore its properties today. One of the more dramatic experiments we did in the two-level condensate was the creation, via a sort of wave-function engineering, of a quantized vortex. In this experiment we made use of both aspects of the two-level system - the distinguishable fluids and the spinor gas. Starting with a near-spherical ball of atoms, all in the lower spin state, we applied the two-photon drive for about $100 \mathrm{~ms}$. At the same time, we illuminated the atoms with an offresonant laser beam whose intensity varied both in time and in space. The laser beam was sufficiently far from resonance that by itself it did not cause the condensate to transition from state to state, but the associated ac Stark shift was large enough to affect the resonant properties of the two-photon drive. The overall scheme is described by Matthews et al. (1999a) and Williams and Holland (1999). The net effect was to leave the atoms near the center of the ball of atoms essentially unperturbed, while converting the population in an equatorial belt around the ball into the upper spin state. This conversion process also imposed a winding in the quantum phase, from 0 around to two pi, in such a way that by the time the drive was turned off, the upper-spin-state atoms were in a vortex state, with a single quantum of circulation (see Fig. 11). The central atoms were nonrotating and, like the pimento in a stuffed olive, served only to mark the location of the vortex core. The core atoms could in turn be selectively blasted away, leaving the upper-state atoms in a bare vortex configuration, whose dynamic properties were shown by postdoc Brian Anderson and grad student Paul Haljan to be essentially the same as those of the filled vortex (Anderson et al., 2000).

\section{Coherence and condensate decay}

One of our favorite BEC experiments was simply to look at how a condensate goes away (Burt et al., 1997). The attraction of this experiment is its inherent simplicity combined with the far-reaching implications of the results. Although it was well established that condensates lived for a finite period, fractions of a second to many seconds depending on conditions, no one had identified the actual process by which atoms were being lost from the condensate. To do this our co-workers Chris Myatt, Rich Ghrist, and Eric Burt simply made condensates and carefully watched the number of atoms and shape of the condensate as a function of time. From these data we determined that the loss process varied with the cube of the density, and hence must be threebody recombination. This was rather what we had expected, but it was nice to have it confirmed. In the process of this measurement we also determined the threebody rate constant, and this was more interesting. Although three-body rate constants still cannot be accurately calculated, it was predicted long ago (Kagan et al., 1985) that they should depend on the coherence properties of the wave function. In a normal thermal sample there are fluctuations and the three-body recombination predominantly takes place at high-density fluctuations. If there is higher-order coherence, however, as one has in macroscopically occupied quantum states such as a 


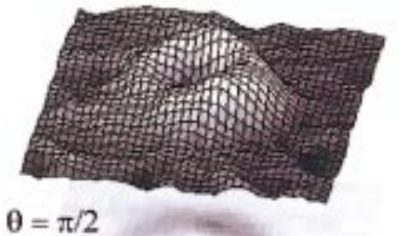

(a)

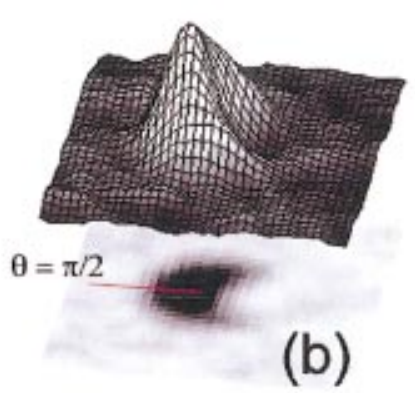

(b)

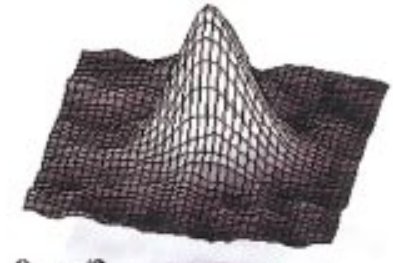

(c)
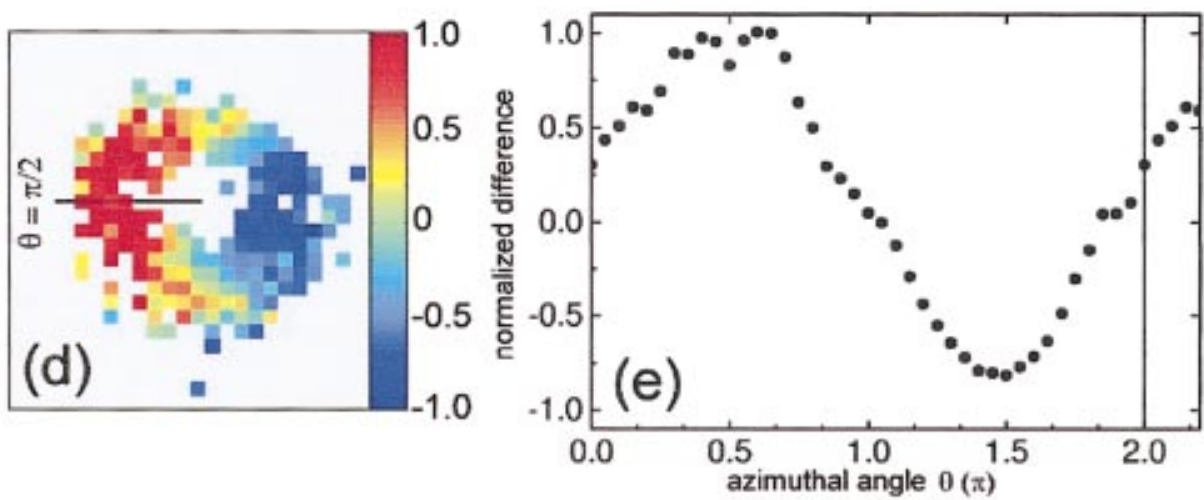

FIG. 11. Condensate images showing the first BEC vortex and the measurement of its phase as a function of azimuthal angle: (a) the density distribution of atoms in the upper hyperfine state after atoms have been put in that state in a way that forms a vortex; (b) the same state after a pi/2 pulse has been applied that mixes upper and lower hyperfine states to give an interferogram reflecting the phase distribution of the upper state; (c) residual condensate in the lower hyperfine state from which the vortex was formed that interferes with a to give the image shown in (b); (d) a color map of the phase difference reflected in (b); (e) radial average at each angle around the ring in (d). The data are repeated after the azimuthal angle $2 \pi$ to better show the continuity around the ring. This shows that the cloud shown in (a) has the $2 \pi$ phase winding expected for a quantum vortex with one unit of angular momentum. From Matthews et al., 1999a [Color].
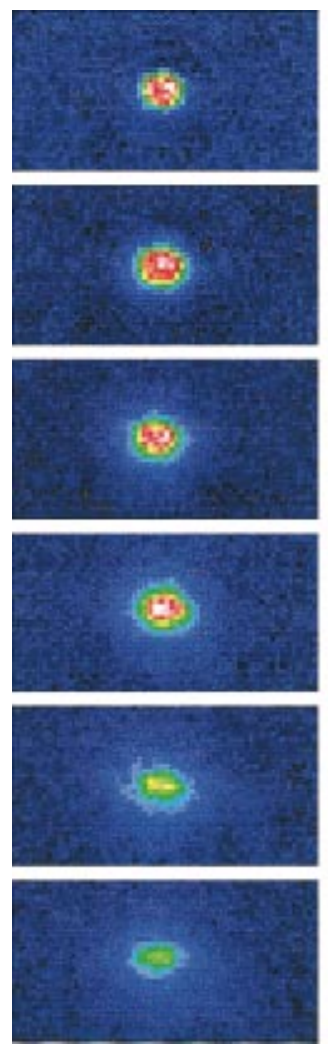
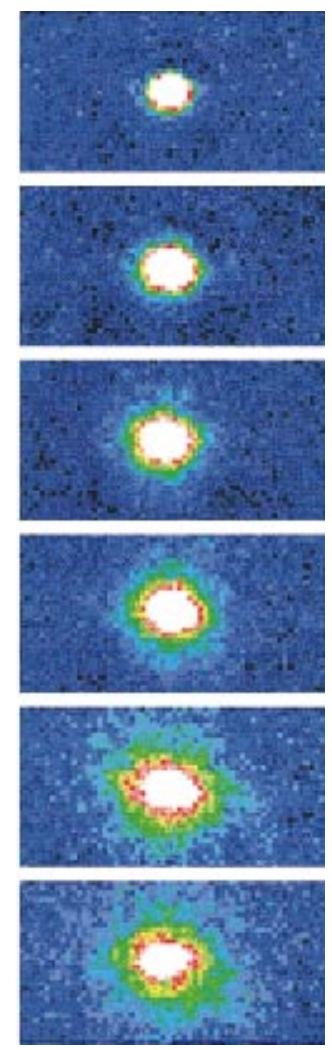

FIG. 12. Bosenova explosion from Roberts et al. (2001). From top to bottom these images show the evolution of the cloud from 0.2 to $4.8 \mathrm{~ms}$ after the interaction was made negative, triggering a collapse. On the left the explosion products are visible as a blue glow expanding out of the center, leaving a small condensate remnant that is unchanged at subsequent times. On the right is the same image amplified by a factor of 3 to better show the $200 \mathrm{nK}$ explosion products [Color]. 
single-mode laser, or as was predicted to exist in a dilute gas BEC, there should be no such density fluctuations. On this basis it was predicted that the three-body rate constant in a Bose-Einstein condensate would be 3 factorial or 6 times lower than what it would be for the same atoms in a thermal sample. It is amusing that such a relatively mundane collision process can be used to probe the quantum correlations and coherence in this fashion. After measuring the three-body rate constant in the condensate we then repeated the measurement in a very cold but uncondensed sample. The predicted factor of 6 (actually 7.4 2.6) was observed, thereby confirming the higher-order coherence of BEC (Burt et al., 1997).

\section{Feshbach resonance physics}

In 1992 Eric Cornell and Chris Monroe realized that dipole collisions at ultralow temperatures might have interesting dependencies on magnetic field, as discussed in the Appendix. With this in mind we approached Boudwijn Verhaar about calculating the magnetic-field dependencies of collisions between atoms in the lower F spin states. When he did this calculation he discovered (Tiesinga et al., 1993) that there were dramatic resonances in all the cross sections as a function of magnetic field that are now known as Feshbach resonances because of their similarity to scattering resonances described by Herman Feshbach in nuclear collisions. From the beginning Verhaar appreciated that these resonances would allow one to tune the $s$-wave scattering length of the atoms and thereby change both the elastic collision cross sections and the self-interaction in a condensate, although this was several years before condensates had been created.

In 1992 we hoped that these Feshbach resonances would give us a way to create enormous elastic collision cross sections that would facilitate evaporative cooling. With this in mind we attempted to find Feshbach resonances in the elastic scattering of first cesium and then, with postdoc Nate Newbury, rubidium. These experiments did provide us with elastic scattering cross sections (Monroe et al., 1993; Newbury et al., 1995), but were unable to locate the few-gauss-wide Feshbach resonances in the thousand-gauss range spanned by then theoretical uncertainty.

By 1997 the situation had dramatically changed, however. A large amount of work on cold collisions, BEC properties, and theoretical advances provided accurate values for the interaction potentials, and so we were fairly confident that there was likely to be a reasonably wide Feshbach resonance in rubidium 85 that was within 20 or 30 gauss of $150 \mathrm{G}$. This was a quite convenient bias field at which to operate our baseball magnetic trap, so we returned to the Feshbach resonance in the hope that we could now use it to make a Bose-Einstein condensate with adjustable interactions.

The time was clearly ripe for Feshbach resonance physics. Within a year Ketterle (Inouye et al., 1998) saw a resonance in sodium through enhanced loss of BEC, Dan Heinzen (Courteille et al., 1998) detected a Feshbach resonance in photoassociation in ${ }^{85} \mathrm{Rb}$, we (Rob- erts et al., 1998; notably students Jake Roberts and Neil Claussen) detected the same resonance in the elastic scattering cross section, and Chu (Vuletic et al., 1999) detected Feshbach resonances in cesium. Our expectations that it would be as easy or easier to form BEC in ${ }^{85} \mathrm{Rb}$ as it was in ${ }^{87} \mathrm{Rb}$ and then use this resonance to manipulate the condensate were sadly naive, however. Due to enhancement of bad collisions by the Feshbach resonance, it was far more difficult and could only be accomplished by following a complicated and precarious evaporation path. However, by finding the correct path and cooling to $3 \mathrm{nK}$ we were able to obtain pure ${ }^{85} \mathrm{Rb}$ condensates of 16000 atoms (Roberts et al., 2001).

The scattering length of these condensates could then be readily adjusted by varying the magnetic field over a few gauss in the vicinity of the Feshbach resonance (Cornish et al., 2000). This has opened up a wide range of possible experiments, from studying the instability of condensates when the self-interaction is sufficiently attractive (negative $a$ ) to exploring the development of correlations in the wave function as the interactions are made large and repulsive. This regime provides one with a new way to probe such disparate subjects as molecular Bose-Einstein condensates and the quantum behavior of liquids, where there is a high degree of correlation. This work represents some of the most recent BEC experiments, but almost everything we have explored with this system has shown dramatic and unexpected results. Thus it is clear that we are far from exhausting the full range of interesting experiments that are yet to be carried out with $\mathrm{BEC}$.

In the first of these Feshbach resonance experiments our students Jake Roberts, Neil Claussen, and postdoc Simon Cornish suddenly changed the magnetic field to make $a$ negative. We observed that, as expected, the condensate became unstable and collapsed, losing a large number of atoms (Roberts et al., 2001). The dynamics of the collapse process were quite remarkable. The condensate was observed to shrink slightly and then undergo an explosion in which a substantial fraction of the atoms were blown off (Donley, 2001). A large fraction of the atoms also simply vanished, presumably turning into undetectable molecules or very energetic atoms, and finally a small cold stable remnant was left behind after the completion of the collapse. This process is illustrated in Fig. 12. Because of its resemblance (on a vastly lower energy scale) to a core collapse supernova, we have named this the Bosenova. There is now considerable theoretical effort to model this process and progress is being made. However, as yet there is no clear explanation of the energy and anisotropy of the atoms in the explosion, the fraction of vanished atoms, and the size of the cold remnant. One of the more puzzling aspects is that the cold remnant can be far larger than the condensate stability condition that determines the collapse point would seem to allow (Donley, 2001).

Another very intriguing result of Feshbach resonance studies in ${ }^{85} \mathrm{Rb}$ was observed when our students Neil Claussen and Sarah Thompson and postdoc Elizabeth Donley quickly jumped the magnetic field close to the 
resonance while keeping the scattering length positive. They found that they could observe the sample oscillate back and forth between being an atomic and a molecular condensate as a function of time after the sudden perturbation (Donley et al., 2002). This curious system of a quantum superposition of two chemically distinct species will no doubt be a subject of considerable future study.

\section{An optimistic appendix}

Until a new technology comes along to replace evaporative cooling, the crucial issue in creating BEC with a new atom is collisions. In practice, this means that planning a BEC experiment with a new atom requires learning to cope with ignorance. It is easy to forget that essentially nothing is known about the ultralowtemperature collisional properties of any atomic or molecular species that is not an atom in the first row of the Periodic Table. One cannot expect theorists to relieve one's ignorance. Interatomic potentials derived from room-temperature spectroscopy are generally not adequate to allow theoretical calculations of cold elastic and inelastic collision rates, even at the order-ofmagnitude level. Although the cold collisional properties of a new atom can be determined, this is a major endeavor, and in most cases it is easier to discover whether evaporation will work by simply trying it.

Launching into such a major new project without any assurances of success is a daunting prospect, but we believe that, if one works hard enough, the probability that any given species can be evaporatively cooled to the point of BEC is actually quite high. The scaling arguments presented below in support of this assertion are largely the same as those that originally encouraged us to pursue BEC in alkalis, although with a bit more refinement provided by age and experience.

Although there is an extensive literature now on evaporative cooling, the basic requirement is simply that there be on the order of 100 elastic collisions per atom per lifetime of the atoms in the trap. Since the lifetime of the atoms in the trap is usually limited by collisions, the requirement can be restated: the rate of elastic collisions must be about two orders of magnitude higher than the rate of bad collisions. As mentioned above, there are three bad collisional processes, and these each have different dependencies on atomic density in the trap, $n$ : background collisions (independent of $n$ ), twobody dipolar relaxation $(\alpha n)$, and three-body recombination $\left(\alpha n^{2}\right)$. The rate for elastic collisions is $n \sigma v$, where $n$ is the mean density, $\sigma$ is the zero-energy $s$-wave cross section, and $v$ is the mean relative velocity. The requirement of 100 elastic-to-inelastic collisions must not only be satisfied immediately after the atoms are loaded into the trap, but also as evaporation proceeds toward larger $n$ and smaller $v$. With respect to evaporating rubidium 87 or the lower hyperfine level of sodium 23, Nature has been kind. One need only arrange for the initial trapped cloud to have sufficiently large $n$, and design a sufficiently low-pressure vacuum chamber, and evaporation works. The main point of this section, however, is that evaporation is likely to be possible even with less favorable collision properties.

Considering the trap loss processes in order, first examine background loss. Trap lifetimes well in excess of what are needed for ${ }^{87} \mathrm{Rb}$ and $\mathrm{Na}$ have been achieved with standard vacuum technology. For example, we now have magnetic trap lifetimes of nearly $1000 \mathrm{~s}$. (This was a requirement to achieve BEC in ${ }^{85} \mathrm{Rb}$ with its less favorable collisions.) If one is willing to accept the added complications of a cryogenic vacuum system, essentially infinite lifetimes are possible. If the background trap loss is low enough to allow evaporative cooling to begin, it will never be a problem at later stages of evaporation because $n v$ increases.

If dipolar relaxation is to be a problem, it will likely be late in the evaporative process when the density is high and velocity low. There is no easy solution to a large dipolar relaxation rate in terms of changing the spring constant of the trap or the pressure of the vacuum chamber. Fortunately, one is not required to accept the value of dipolar collisions that Nature provides. In fact, all one really has to do is operate the trap with a very low magnetic bias field in a magnetic trap, or if one uses an optical trap very far off-resonance (such as $\mathrm{CO}_{2}$ laser), trap the atoms in the lowest spin state, for which there are no dipole collisions. The bias field dependence comes about because below a field of roughly $5 \mathrm{G}$, the dipolar rate in the lower hyperfine level drops rapidly to zero. This behavior is simple to understand. At low temperature, the incoming collisional channel must be purely $s$ wave. Dipolar relaxation changes the projection of spin angular momentum, so to conserve angular momentum the outgoing collisional channel must be $d$ wave or higher. The nonzero outgoing angular momentum means that there is an angular momentum barrier in the effective molecular potential, a barrier of a few hundred microkelvin. If the atoms are trapped in the lower hyperfine state $\left(F=1, m_{F}=-1\right.$, in rubidium 87$)$ the outgoing energy from a dipolar collision is only the Zeeman energy in the trapping fields, and for B less than about 5 $G$ this energy is insufficient to get the atoms back out over the angular momentum barrier. If relaxation is to occur, it can happen only at interatomic radii larger than the outer turning point of the angular momentum barrier. For smaller and smaller fields, the barrier gets pushed further out, with correspondingly lower transition rates.

It is unlikely that the three-body recombination rate constant could ever be so large that three-body recombination would be a problem when the atoms are first loaded from a MOT into the evaporation trap. As evaporation proceeds, however, just as for the dipolar collisions, it becomes an increasingly serious concern. Because of its density dependence, however, it can always be avoided by manipulating the trapping potential. Adiabatically reducing the trap confinement has no effect on the phase-space density but it reduces both the density and the atom velocity. The ratio of three-body to elastic collisions scales as $1 / n v$. Therefore, as long as 
one can continue to turn down the confining strength of one $s$ trap, one can ensure that three-body recombination will not prevent evaporative cooling all the way down to the BEC transition.

To summarize, given (i) a modestly flexible magnetic trap, (ii) an arbitrarily good vacuum, (iii) a true ground state with $F \neq 0$, and (iv) nonpathological collisional properties, almost any magnetically trappable species can be successfully evaporated to BEC. If one is using a very far off-resonance optical trap (such as a $\mathrm{CO}_{2}$ dipole trap) one can extend these arguments to atoms that cannot be magnetically trapped. In that case, however, current technology makes it more difficult to optimize the evaporation conditions than in magnetic traps, and the requirement to turn the trap down sufficiently to avoid a large three-body recombination rate can be more difficult. Nevertheless, one can plausibly look forward to $\mathrm{BEC}$ in a wide variety of atoms and molecules in the future.

\section{ACKNOWLEDGMENTS}

We acknowledge support from the National Science Foundation, the Office of Naval Research, and the National Institute of Standards and Technology. We have benefited enormously from the hard work and intellectual stimulation of our many students and postdocs. They include Brian Anderson, Mike Anderson, Steve Bennett, Eric Burt, Neil Claussen, Ian Coddington, Kristan Corwin, Liz Donley, Peter Engels, Jason Ensher, David Hall, Debbie Jin, Tetsuo Kishimoto, Heather Lewandowski, Mike Matthews, Jeff McGuirk, Chris Monroe, Chris Myatt, Nate Newbury, Scott Papp, Cindy Regal, Mike Renn, Jake Roberts, Peter Schwindt, David Sesko, Michelle Stephens, William Swann, Sarah Thompson, Thad Walker, Yingju Wang, Richard Watts, Chris Wood, and Josh Zirbel. We also have had help from many other JILA faculty, including John Bohn, Chris Greene, and Murray Holland.

\section{REFERENCES}

Abraham, E. R. I., W. I. McAlexander, C. D. Sackett, and R. G. Hulet, 1995, Phys. Rev. Lett. 74, 1315.

Anderson, M. H., J. R. Ensher, M. R. Matthews, C. E. Wieman, and E. A. Cornell, 1995, Science 269, 198.

Anderson, M. H., J. R. Ensher, M. R. Matthews, C. E. Wieman, and E. A. Cornell, 1996, in Laser Spectroscopy, XII International Conference, edited by Massimo Inguscio, Maria Allegrini, and Antonio Sasso (World Scientific, Singapore), p. 3.

Anderson, B. P., P. C. Haljan, C. E. Wieman, and E. A. Cornell, 2000, Phys. Rev. Lett. 85, 2857.

Anderson, M. H., W. Petrich, J. R. Ensher, and E. A. Cornell, 1994, Phys. Rev. A 50, R3597.

Andreev, S. V., V. I. Balykin, V. S. Letokhov, and V. G. Minogin, 1981, Zh. Eksp. Teor. Fiz. 34, 463.

Arimondo, E., W. D. Phillips, and F. Strumia, 1991, Eds., Proceedings of the International School of Physics "Enrico Fermi," Course CXVIII, Laser Manipulation of Atoms and Ions (North-Holland, Amsterdam).
Bagnato, V. S., G. P. Lafyatis, A. G. Martin, E. L. Raab, R. N. Ahmad-Bitar, and D. E. Pritchard, 1987, Phys. Rev. Lett. 58, 2194.

Bell, D. A., F. H. Hess, G. P. Kochanski, S. Buchman, L. Pollack, Y. M. Xiao, D. Kleppner, and T. J. Greytak, 1986, Phys. Rev. B 34, 7670.

Bjorkholm, J. E., R. R. Freeman, A. Ashkin, and D. B. Pearson, 1978, Phys. Rev. Lett. 41, 1361.

Bose, S., 1924, Z. Phys. 26, 178.

Burt, E. A., R. W. Ghrist, C. J. Myatt, M. J. Holland, E. A. Cornell, and C. E. Wieman, 1997, Phys. Rev. Lett. 79, 337.

Chu, S., 1998, Rev. Mod. Phys. 70, 685.

Chu, S., J. E. Bjorkholm, A. Ashkin, and A. Cable, 1986, Phys. Rev. Lett. 57, 314.

Chu, S., L. Holberg, J. E. Bjorkholm, A. Cable, and A. Ashkin, 1985, Phys. Rev. Lett. 55, 48.

Chu, S., D. S. Weiss, Y. Shevy, and P. J. Ungar, 1989, Proceedings 11th International Conference on Atomic Physics (World Scientific, Singapore), pp. 636-638.

Cohen-Tannoudji, C. N., 1998, Rev. Mod. Phys. 70, 707.

Cornell, E. A., J. R. Ensher, and C. E. Wieman, 1999, in Proceedings of the International School of Physics "Enrico Fermi," Course CXL, edited by M. Inguscio, S. Stringari, and C. E. Wieman (Italian Physical Society, Bologna), p. 15. Also available as cond-mat/9903109.

Cornell, E. A., D. S. Hall, M. R. Matthews, and C. E. Wieman, 1998, J. Low Temp. Phys. 113, 151.

Cornell, E. A., C. Monroe, and C. E. Wieman, 1991, Phys. Rev. Lett. 67, 2439.

Cornish, S. L., N. R. Claussen, J. L. Roberts, E. A. Cornell, and C. E. Wieman, 2000, Phys. Rev. Lett. 85, 1795.

Courteille, P., R. S. Freeland, D. J. Heinzen, F. A. vanAbeelen, and B. J. Verhaar, 1998, Phys. Rev. Lett. 81, 69.

Dalfovo, F., S. Giorgini, L. P. Pitaevskii, and S. Stringari, 1999, Rev. Mod. Phys. 71, 463.

Dalibard, J., and C. Cohen-Tannoudji, 1989, J. Opt. Soc. Am. B 6, 2023.

Dalibard, J., C. Salomon, A. Aspect, E. Arimondo, R. Kaiser, N. Vansteenkiste, and C. Cohen-Tannoudji, 1989, Proceedings 11th International Conference on Atomic Physics (World Scientific, Singapore), pp. 199-214.

Dodd, R. J., M. Edwards, C. W. Clark, and K. Burnett, 1998, Phys. Rev. A 57, R32.

Donley, E. A., 2001, Nature (London) 412, 295.

Donley, E. A., N. R. Claussen, S. T. Thompson, and C. E. Wieman, 2002, Nature (London) 417, 529.

Doyle, J. M., 1991a, Phys. Rev. Lett. 67, 603.

Doyle, J. M., 1991b, Ph.D. thesis (Massachusetts Institute of Technology).

Einstein, A., 1924, Sitzungsber. K. Preuss. Akad. Wiss., Phys. Math. K1. 261.

Einstein, A., 1925, Sitzungsber. K. Preuss. Akad. Wiss., Phys. Math. Kl. 3.

Ertmer, W., R. Blatt, J. L. Hall, and M. Zhu, 1985, Phys. Rev. Lett. 54, 996.

Fedichev, P. O. and G. V. Shlyapnikov, 1998, Phys. Rev. A 58, 3146.

Fedichev, P. O., G. V. Shlyapnikov, and J. T. M. Walraven, 1998, Phys. Rev. Lett. 80, 2269.

Gardner, J. R., R. A. Cline, J. D. Miller, D. J. Heinzen, H. M. J. M. Boesten, and B. J. Verhaar, 1995, Phys. Rev. Lett. 74, 3764 . 
Gibble, K., S. Chang, and R. Legere, 1995, Phys. Rev. Lett. 75, 2666.

Gillaspy, J. D., I. F. Silvera, and J. S. Brooks, 1989, Phys. Rev. B 40, 210.

Hall, D. S., M. R. Matthews, J. R. Ensher, C. E. Wieman, and E. A. Cornell, 1998a, Phys. Rev. Lett. 81, 1539.

Hall, D. S., M. R. Matthews, C. E. Wieman, and E. A. Cornell, 1998b, Phys. Rev. Lett. 81, 1543.

Hänsch, T. W., and A. L. Schawlow, 1975, Opt. Commun. 13, 68.

Hardy, W. N., M. Morrow, R. Jochemsen, and A. Berlinsky, 1982, Physica B \& C 110, 1964.

Hecht, C. E., 1959, Physica 25, 1159.

Hess, H. F., 1986, Phys. Rev. B 34, 3476.

Hess, H. F., D. A. Bell, G. P. Kochanski, R. W. Cline, D. Kleppner, and T. J. Greytak, 1983, Phys. Rev. Lett. 51, 483.

Hutchinson, D. A. W., E. Zaremba, and A. Griffin, 1997, Phys. Rev. Lett. 78, 1842.

Inouye, S., M. R. Andrews, J. Stenger, H. J. Miesner, D. M. Stamper-Kurn, and W. Ketterle, 1998, Nature (London) 392, 151.

Jackson, B., and E. Zaremba, 2002, Phys. Rev. Lett. 88, 180402. Jin, D. S., J. R. Ensher, M. R. Matthews, C. E. Wieman, and E. A. Cornell, 1996, Phys. Rev. Lett. 77, 420.

Jin, D. S., M. R. Matthews, J. R. Ensher, C. E. Wieman, and E. A. Cornell, 1997, Phys. Rev. Lett. 78, 764.

Johnson, B. R., J. S. Denker, N. Bigelow, L. P. Levy, J. H. Freed, and D. M. Lee, 1984, Phys. Rev. Lett. 52, 1508.

Kagan, Y., G. V. Shlyapnikov, and I. A. Vartanyants, 1984, Phys. Lett. A 101, 27.

Kagan, Y., B. V. Svistunov, and G. V. Shlyapnikov, 1985, JETP Lett. 42, 209.

Kagan, Y., I. A. Vartanyants, and G. V. Shlyapnikov, 1981, Sov. Phys. JETP 54, 590.

Ketterle, W., K. B. Davis, M. A. Joffe, A. Martin, and D. E. Pritchard, 1993, Phys. Rev. Lett. 70, 2253.

Ketterle, W., D. S. Durfee, and D. M. Stamper-Kurn, 1999, in Proceedings of the International School of Physics "Enrico Fermi," Course CXL, edited by M. Inguscio, S. Stringari, and C. E. Wieman (Italian Physical Socity, Bologna), p. 176.

Lagendijk, A., I. F. Silvera, and B. J. Verhaar, 1986, Phys. Rev. B 33, 626.

Letokhov, V., 1968, Zh. Eksp. Teor. Fiz. Pis'ma Red 7, 348.

Lett, P. D., K. Helmerson, W. D. Phillips, L. P. Ratliff, S. L. Rolston, and M. E. Wagshul, 1993, Phys. Rev. Lett. 71, 2200. Lett, P. D., R. N. Watts, C. I. Westbrook, W. D. Phillips, P. L. Gould, and H. J. Metcalf, 1988, Phys. Rev. Lett. 61, 169.

Liu, W. V., 1997, Phys. Rev. Lett. 79, 4056.

London, F., 1938, Nature (London) 141, 643.

Lovelace, R. V. E., C. Mehanian, T. J. Tomilla, and D. M. Lee, 1985, Nature (London) 318, 30.

Lovelace, R. V. E., and T. J. Tommila, 1987, Phys. Rev. A 35, 3597.

Luiten, O. J., H. G. C. Werij, I. D. Setija, M. W. Reynolds, T. W. Hijmans, and J. T. M. Walraven, 1993, Phys. Rev. Lett. 70, 544.

Majorana, E., 1931, Nuovo Cimento 8, 107.

Martin, A. G., K. Helmerson, V. S. Bagnato, G. P. Lafyatis, and D. E. Pritchard, 1988, Phys. Rev. Lett. 61, 2431.

Matthews, M. R., B. P. Anderson, P. C. Haljan, D. S. Hall, M. J. Holland, J. E. Williams, C. E. Wieman, and E. A. Cornell, 1999b, Phys. Rev. Lett. 83, 3358.
Matthews, M. R., B. P. Anderson, P. C. Haljan, D. S. Hall, C. E. Wieman, and E. A. Cornell, 1999a, Phys. Rev. Lett. 83, 2498.

Matthews, M. R., D. S. Hall, D. S. Jin, J. R. Ensher, C. E. Wieman, E. A. Cornell, F. Dalfovo, C. Minniti, and S. Stringari, 1998, Phys. Rev. Lett. 81, 243.

McAlexander, W. I., E. R. I. Abraham, N. W. M. Ritchie, C. J. Williams, H. T. C. Stoof, and R. G. Hulet, 1995, Phys. Rev. A 51, R871.

Migdall, A. L., J. V. Prodan, W. D. Phillips, T. H. Bergeman, and H. J. Metcalf, 1985, Phys. Rev. Lett. 54, 2596.

Miller, J. D., R. A. Cline, and D. J. Heinzen, 1993, Phys. Rev. Lett. 71, 2204.

Monroe, C., 1992, Ph.D. thesis (University of Colorado, Boulder).

Monroe, C., W. Swann, H. Robinson, and C. Wieman, 1990, Phys. Rev. Lett. 65, 1571.

Monroe, C. R., E. A. Cornell, C. A. Sackett, C. J. Myatt, and C. E. Wieman, 1993, Phys. Rev. Lett. 70, 414.

Myatt, C. J., E. A. Burt, R. W. Ghrist, E. A. Cornell, and C. E. Wieman, 1997, Phys. Rev. Lett. 78, 586.

Myatt, C. J., N. R. Newberry, R. W. Ghrist, S. Loutzenhiser, and C. E. Wieman, 1996, Opt. Lett. 21, 290.

Neuhauser, W., M. Hohenstatt, P. Toschek, and H. Dehmelt, 1978, Phys. Rev. Lett. 41, 233.

Newbury, N. R., C. J. Myatt, and C. E. Wieman, 1995, Phys. Rev. A 51, R2680.

Pais, A., 1982, Subtle Is the Lord .. (Oxford University Press, Oxford), English translation of Einstein's quotes and historical interpretation.

Petrich, W., M. H. Anderson, J. R. Ensher, and E. A. Cornell, 1994, J. Opt. Soc. Am. B 11, 1332.

Petrich, W., M. H. Anderson, J. R. Ensher, and E. A. Cornell, 1995, Phys. Rev. Lett. 74, 3352.

Phillips, W. D., 1998, Rev. Mod. Phys. 70, 707.

Pritchard, D. E., 1986, in Electronic and Atomic Collisions, edited by D. C. Lorents, W. Meyerhof, and J. R. Peterson (North-Holland, Amsterdam), p. 593.

Pritchard, D. E., K. Helmerson, and A. G. Martin, 1989, Proceedings 11th International Conference on Atomic Physics (World Scientific, Singapore), pp. 179-197.

Pritchard, D. E., E. L. Raab, V. Bagnato, C. E. Wieman, and R. N. Watts, 1986, Phys. Rev. Lett. 57, 310.

Prodan, J., A. Migdall, W. D. Phillips, I. So, H. Metcalf, and J. Dalibard, 1985, Phys. Rev. Lett. 54, 992.

Raab, E. L., M. Prentiss, A. Cable, S. Chu, and D. E. Pritchard, 1987, Phys. Rev. Lett. 59, 2631.

Roberts, J. L., N. R. Claussen, J. P. Burke, Jr., C. H. Greene, E. A. Cornell, and C. E. Wieman, 1998, Phys. Rev. Lett. 81, 5109.

Roberts, J. L., N. R. Claussen, S. L. Cornish, E. A. Donley, E. A. Cornell, and C. E. Wieman, 2001, Phys. Rev. Lett. 86, 4211.

Rohner, H., 1994, Proceedings of the 39th Symposium on the Art of Glassblowing (American Scientific Glassblowing Society, Wilmington, Delaware), p. 57.

Sesko, D., T. Walker, C. Monroe, A. Gallagher, and C. Wieman, 1989, Phys. Rev. Lett. 63, 961.

Sesko, D., T. G. Walker, and C. E. Wieman, 1991, J. Opt. Soc. Am. B 8, 946.

Silvera, I. F., and J. T. M. Walraven, 1980, Phys. Rev. Lett. 44, 164. 
Sprik, R., J. T. M. Walraven, and I. F. Silvera, 1985, Phys. Rev. B 32, 5668.

Stoof, H. T. C., 2000, private communication.

Stwalley, W. C., and L. H. Nosanow, 1976, Phys. Rev. Lett. 36, 910.

Thorsheim, H. R., J. Weiner, and P. S. Julienne, 1987, Phys. Rev. Lett. 58, 2420.

Tiesinga, E., B. J. Verhaar, and H. T. C. Stoof, 1993, Phys. Rev. A 47, 4114.

Tisza, L., 1938, Nature (London) 141, 913.

Vuletic, V., A. J. Kerman, C. Chin, and S. Chu, 1999, Phys.
Rev. Lett. 82, 1406.

Walker, T., D. Sesko, and C. Wieman, 1990, Phys. Rev. Lett. 64, 408.

Watts, R. N., and C. E. Wieman, 1986, Opt. Lett. 11, 291.

Williams, J. E., and M. J. Holland, 1999, Nature (London) 401, 568.

Wineland, D. J., and H. Dehmelt, 1975, Bull. Am. Phys. Soc. 20, 637.

Wineland, D. J., R. E. Drullinger, and F. L. Walls, 1978, Phys. Rev. Lett. 40, 1639. 